\title{
Discovery of a hot, transiting, Earth-sized planet and a second temperate, non-transiting planet around the M4 dwarf GJ 3473 (TOI-488)^
}

\author{
J. Kemmer ${ }^{1, \star \star}$, S. Stock ${ }^{1, \star \star}$, D. Kossakowski ${ }^{2, \star \star}$, A. Kaminski ${ }^{1}$, K. Molaverdikhani ${ }^{1,2}$, M. Schlecker ${ }^{2, \star \star,}$ \\ J. A. Caballero ${ }^{3}$, P. J. Amado ${ }^{4}$, N. Astudillo-Defru ${ }^{5}$, X. Bonfils ${ }^{6}$, D. Ciardi ${ }^{7}$, K. A. Collins 8 , N. Espinoza ${ }^{9}$, \\ A. Fukui ${ }^{10,11}$, T. Hirano ${ }^{12}$, J. M. Jenkins ${ }^{13}$, D. W. Latham ${ }^{8}$, E. C. Matthews ${ }^{14}$, N. Narita ${ }^{15,16,17,18}$, E. Palléle,11, \\ H. Parviainen ${ }^{18,11}$, A. Quirrenbach ${ }^{1}$, A. Reiners ${ }^{19}$, I. Ribas ${ }^{20,21}$, G. Ricker ${ }^{14}$, J. E. Schlieder ${ }^{22}$, S. Seager ${ }^{14,23,24}$, \\ R. Vanderspek ${ }^{14}$, J. N. Winn ${ }^{25}$, J. M. Almenara ${ }^{6}$, V. J. S. Béjarr ${ }^{18,11}$, P. Bluhm ${ }^{1, \star \star}$, F. Bouchy ${ }^{26}$, P. Boyd ${ }^{22}$, \\ J. L. Christiansen ${ }^{7}$, C. Cifuentes ${ }^{3}$, R. Cloutier ${ }^{8}$, K. I. Collins ${ }^{27}$, M. Cortés-Contreras ${ }^{3}$, I. J M. Crossfield ${ }^{14}$, \\ N. Crouzet ${ }^{28}$, J. P. de Leon ${ }^{29}$, D. D. Della-Rose ${ }^{30}$, X. Delfosse ${ }^{6}$, S. Dreizler ${ }^{19}$, E. Esparza-Borges ${ }^{11}$, Z. Essack ${ }^{23,24}$, \\ Th. Forveille ${ }^{6}$, P. Figueira ${ }^{31,32}$, D. Galadí-Enríquez ${ }^{33}$, T. Gan ${ }^{34}$, A. Glidden ${ }^{23,14}$, E. J. Gonzales ${ }^{35, \star \star \star}$, P. Guerra ${ }^{36}$, \\ H. Harakawa ${ }^{37}$, A. P. Hatzes ${ }^{38}$, Th. Henning ${ }^{2}$, E. Herrero ${ }^{21}$, K. Hodapp ${ }^{39}$, Y. Hori ${ }^{17,40}$, S. B. Howell ${ }^{13}$, M. Ikoma ${ }^{10}$, \\ K. Isogai ${ }^{41}$, S. V. Jeffers ${ }^{19}$, M. Kürster ${ }^{2}$, K. Kawauchi ${ }^{10}$, T. Kimura ${ }^{10}$, P. Klagyivik ${ }^{18,11,42}$, T. Kotani ${ }^{17,40,43}$, \\ T. Kurokawa ${ }^{40,44}$, N. Kusakabe ${ }^{17,40}$, M. Kuzuhara ${ }^{17,40}$, M. Lafarga ${ }^{20,21}$, J. H. Livingston ${ }^{29}$, R. Luque ${ }^{18,11}$, \\ R. Matson ${ }^{45}$, J. C. Morales ${ }^{20,21}$, M. Mori ${ }^{29}$, P. S. Muirhead ${ }^{46}$, F. Murgas ${ }^{18,11}$, J. Nishikawa ${ }^{40,17,43}$, T. Nishiumi ${ }^{43,40}$, \\ M. Omiya ${ }^{17,40}$, S. Reffert ${ }^{1}$, C. Rodríguez López ${ }^{4}$, N. C. Santos ${ }^{32,47}$, P. Schöfer ${ }^{19}$, R. P. Schwarz ${ }^{48}$, B. Shiao ${ }^{9}$,

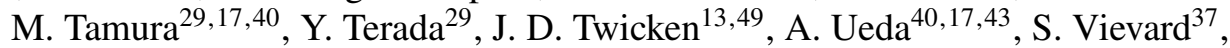 \\ N. Watanabe ${ }^{43,17,40}$, and M. Zechmeister ${ }^{19}$
}

(Affiliations can be found after the references)

Received 18 July 2020 / Accepted 10 September 2020

\begin{abstract}
We present the confirmation and characterisation of GJ 3473 b (G 50-16, TOI-488.01), a hot Earth-sized planet orbiting an M4 dwarf star, whose transiting signal $(P=1.1980035 \pm 0.0000018 \mathrm{~d})$ was first detected by the Transiting Exoplanet Survey Satellite (TESS). Through a joint modelling of follow-up radial velocity observations with CARMENES, IRD, and HARPS together with extensive ground-based photometric follow-up observations with LCOGT, MuSCAT, and MuSCAT2, we determined a precise planetary mass, $M_{\mathrm{b}}=1.86 \pm 0.30 M_{\oplus}$, and radius, $R_{\mathrm{b}}=1.264 \pm 0.050 R_{\oplus}$. Additionally, we report the discovery of a second, temperate, non-transiting planet in the system, GJ $3473 \mathrm{c}$, which has a minimum mass, $M_{\mathrm{c}} \sin i=7.41 \pm 0.91 M_{\oplus}$, and orbital period, $P_{\mathrm{c}}=15.509 \pm 0.033 \mathrm{~d}$. The inner planet of the system, GJ $3473 \mathrm{~b}$, is one of the hottest transiting Earth-sized planets known thus far, accompanied by a dynamical mass measurement, which makes it a particularly attractive target for thermal emission spectroscopy.
\end{abstract}

Key words. planetary systems - planets and satellites: detection - techniques: radial velocities - techniques: photometric stars: late-type - stars: individual: G 50-16

\section{Introduction}

The detection of transiting planets with the radial velocity (RV) method enables us to derive a comprehensive characterisation of their properties. In particular, it permits the measurement of a dynamical planetary mass and, hence, a measurement of the planetary mean density when combined with the planetary radius derived from the transit light curve. From comparisons with theoretical models, the density of a planet provides information about its composition and structure and, therefore, it

\footnotetext{
${ }^{\star} \mathrm{RV}$ data are only available at the CDS via anonymous ftp to cdsarc.u-strasbg.fr $(130.79 .128 .5)$ or via http://cdsarc. u-strasbg.fr/viz-bin/cat/J/A+A/642/A236

${ }^{\star \star}$ Fellow of the International Max Planck Research School for Astronomy and Cosmic Physics at the University of Heidelberg (IMPRS-HD). ${ }^{\star \star \star}$ National Science Foundation Graduate Research Fellow.
}

plays a key role in understanding planet formation and evolution (e.g. Southworth 2010; Marcy et al. 2014; Rogers 2015; Fulton et al. 2017; Bitsch et al. 2019; Zeng et al. 2019). Furthermore, additional non-transiting planets in the system can be detected with the RV method. Such multi-planetary systems hold valuable information because the dynamical interaction between the planets can have a significant influence on their formation and evolution, as well as shaping the currently observed architecture of the system (e.g. Lissauer 2007; Zhu et al. 2012; Anglada-Escudé et al. 2013; Mills \& Mazeh 2017; Morales et al. 2019).

A significant fraction of the over 3000 transiting exoplanets known today ${ }^{1}$ were discovered by the Kepler satellite (Borucki et al. 2010; Borucki 2016). However, Kepler's focus on faint

\footnotetext{
1 On 26 August 2020, 3189 transiting exoplanets were listed by exoplanetarchive.ipac.caltech.edu/
} 
stars $\left(K_{p}>12 \mathrm{mag}\right)$ impeded detailed follow-up studies of those planets using ground-based facilities. In contrast, the Transiting Exoplanet Survey Satellite (TESS; Ricker et al. 2015) is now filling in this gap. To date, TESS has already found more than 50 confirmed transiting planets, and many more candidates, orbiting bright, nearby stars $(G \sim 6-13 \mathrm{mag}, d \sim 10-340 \mathrm{pc})$. One of its level-one science requirements is to measure the masses for 50 transiting planets with radii smaller than $4 R_{\oplus}$ by RV followup observations ${ }^{2}$. What is particularly interesting in this regime are planets that are orbiting $\mathrm{M}$ dwarf stars. The relative transit depth, and thus the detection probability of rocky planets around M dwarfs, is much higher compared to larger stars of earlier spectral types. Still, despite M dwarfs being the most common stars in our Galaxy (e.g. Chabrier 2003; Henry et al. 2006) and the fact that small planets are more abundant around later type stars (Howard et al. 2012; Bonfils et al. 2013; Mulders et al. 2015; Dressing \& Charbonneau 2015; Gaidos et al. 2016; HardegreeUllman et al. 2019), only a few precise dynamical masses of such planets have currently been determined. Prior to the TESS mission, only 12 planets with radii smaller than $R=2 R_{\oplus}$ and dynamical mass measurements to a precision better than $30 \%$ were known to orbit stars with temperatures, $T_{\text {eff }}<4000 \mathrm{~K}$. Thanks to the intensive RV follow-up of TESS planet candidates, this number already increased by seven new planets (see Table A.1 for the full list). The brightness of these cool TESS host stars, combined with their small size, makes many of them ideal targets for atmospheric characterisation by transmission or thermal emission spectroscopy with upcoming space-borne or ground-based instruments (Kempton et al. 2018; Batalha et al. 2018).

Here, we report the discovery of a planetary system around the intermediate M dwarf GJ 3473. The inner, Earth-sized planet was first detected as a transiting planet candidate by TESS. Our extensive RV monitoring campaign, using CARMENES, IRD, and HARPS, confirms its planetary nature and reveals a second, more massive, non-transiting planet on a wider orbit. This paper is structured as follows: Sect. 2 describes the data used in this study. In Sect. 3, the properties of the host star are presented. The analysis of the data is set out in Sect. 4 and the results are discussed in Sect. 5. Finally, we give our conclusions in Sect. 6.

\section{Data}

\subsection{TESS}

GJ 3473 (TIC 452866790) was observed by TESS with a twominute cadence in Sector 7 (Camera \#1, CCD \#3) between 7 January and 2 February 2019 and is listed to have a transiting planet candidate on the TESS releases website (TOI-488.01). Due to its proximity to the ecliptic plane, it will not be observed again by TESS during its primary mission, but will be revisited in Sector 34 of the TESS extended mission in the third year $^{3}$. The time series had a gap between BJD $=2458503.04$ and $\mathrm{BJD}=2458504.71$ because of the data downlink and telescope re-pointing (see Fig. 2). The light curves produced by the Science Processing Operations Center (SPOC; Jenkins et al. 2016) are available on the Mikulski Archive for Space Telescopes ${ }^{4}$. For our analysis, we used the systematics-corrected simple aperture

\footnotetext{
2 https://heasarc.gsfc.nasa.gov/docs/tess/ primary-science.html, visited on 28 June 2020.

3 https://heasarc.gsfc.nasa.gov/cgi-bin/tess/webtess/ wtv . py? Entry=452866790, visited on 28 April 2020.

4 https://mast.stsci.edu
}

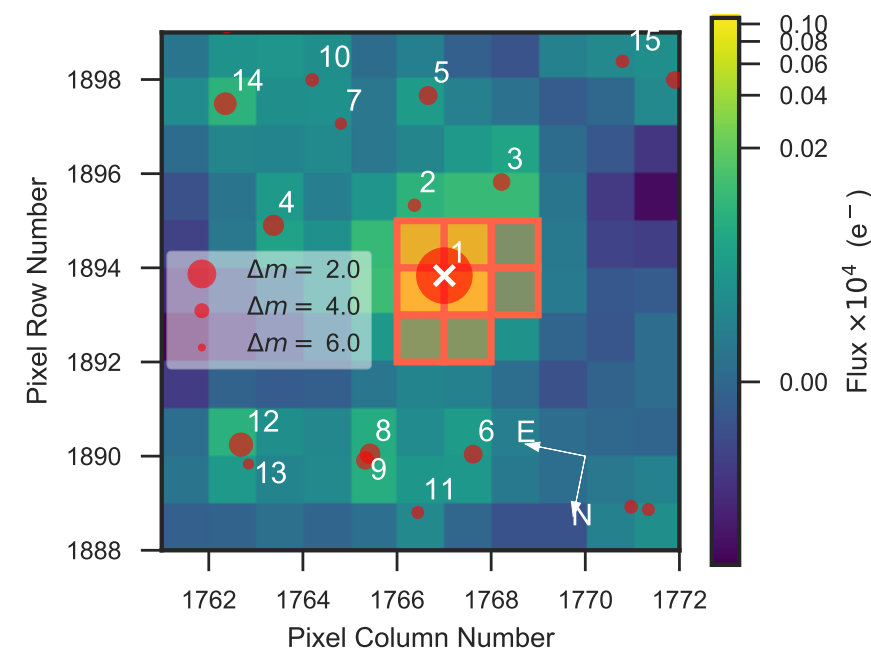

Fig. 1. TESS TPF of GJ 3473. The planet-host star is marked by a white cross and the pixels of the aperture mask used for the retrieval of the light curve are highlighted with orange borders. Sources listed in the Gaia DR2 catalogue (Gaia Collaboration 2018) are indicated by red circles (size proportional to their brightness difference with GJ 3473). Source \#3 is LP 544-12, the common proper motion companion to GJ 3473.

photometry (PDC-SAP) light curve (Smith et al. 2012; Stumpe et al. 2012, 2014). A plot of the target pixel file (TPF) and the aperture mask that is used for the simple aperture photometry (SAP), generated with tpfplotter ${ }^{5}$, is shown in Fig. 1. The TESS data have a median internal uncertainty of $2.35 \mathrm{ppt}$ (parts per thousand) and root mean square (rms) of $2.2 \mathrm{ppt}$ around the mean. See Luque et al. (2019), Dreizler et al. (2020), Nowak et al. (2020), and Bluhm et al. (2020) for further details on the applied methodology.

\subsection{High-resolution spectroscopy}

High-resolution follow-up spectroscopy of the TESS planet candidates is arranged by the TESS follow-up programme (TFOP), "Precise Radial Velocities" SG4 subgroup". The goal is to achieve a full validation of the candidates and to ultimately provide their mass measurement.

CARMENES. As part of the CARMENES guaranteed time observation programme to search for exoplanets around M dwarfs (Reiners et al. 2018), we observed GJ 3473 with CARMENES (Calar Alto high-Resolution search for $M$ dwarfs with Exoearths with Near-infrared and visible Echelle Spectrographs; Quirrenbach et al. 2014). CARMENES is a highresolution spectrograph at the $3.5 \mathrm{~m}$ Calar Alto telescope that consists of two cross-dispersed echelle channels operating in the spectral ranges of 0.52 to $0.96 \mu \mathrm{m}$ in the visible light (VIS, $R=94600$ ) and 0.96 to $1.71 \mu \mathrm{m}$ in the near infrared (NIR, $R=80400$ ), respectively. The observations began at the end of March 2019, just after the announcement of the transiting planet candidate, and ended in January 2020. In this period, we collected 67 pairs of VIS and NIR spectra with exposure times of about 30 min each. Within the standard CARMENES data flow, the spectra are calibrated using CARACAL (Caballero et al. 2016a), while the RVs are calculated using SERVAL (Zechmeister et al. 2018). The RVs are corrected for barycentric motion,

\footnotetext{
5 https://github.com/jlillo/tpfplotter

6 https://tess.mit.edu/followup/
} 
Table 1. Summary of the ground-based transit follow-up observations.

\begin{tabular}{lcccccccc}
\hline \hline Telescope & Date & Transit $^{(a)}$ & Filter & $\begin{array}{c}t_{\text {exp }} \\
{[\mathrm{s}]}\end{array}$ & $\begin{array}{c}\text { Duration } \\
\text { [min] }\end{array}$ & $N_{\text {obs }}$ & $\begin{array}{c}\text { Aperture } \\
\text { [pix] }\end{array}$ & $\begin{array}{c}\text { rms } \\
\text { [ppt] }\end{array}$ \\
\hline LCOGT McD & $2019-03-19$ & 58 & $z_{s}$ & 100 & 234 & 110 & 18 & 1.25 \\
MuSCAT2 & $2019-12-21$ & 290 & $i$ & 30 & 237 & 675 & 32 & 1.75 \\
MuSCAT2 & $2019-12-21$ & 290 & $z_{s}$ & 20 & 237 & 457 & 32 & 1.65 \\
MuSCAT2 & $2020-01-02$ & 300 & $r$ & 18 & 254 & 823 & 32 & 2.45 \\
MuSCAT2 & $2020-01-02$ & 300 & $i$ & 18 & 254 & 845 & 32 & 2.12 \\
MuSCAT2 & $2020-01-02$ & 300 & $z_{s}$ & 18 & 254 & 845 & 32 & 1.60 \\
MuSCAT & $2020-01-18$ & 313 & $r$ & 20 & 202 & 553 & 24 & 1.85 \\
MuSCAT & $2020-01-18$ & 313 & $z_{s}$ & 20 & 202 & 551 & 26 & 1.11 \\
LCOGT CTIO & $2020-02-21$ & 341 & $i_{p}$ & 60 & 224 & 145 & 20 & 1.56 \\
LCOGT CTIO & $2020-02-27$ & 346 & $i_{p}$ & 60 & 229 & 145 & 19 & 1.58 \\
LCOGT SAAO & $2020-03-13$ & 359 & $z_{s}$ & 100 & 230 & 101 & 16 & 1.10 \\
\hline
\end{tabular}

Notes. ${ }^{(a)}$ Transit number after the first transit observed by TESS. ${ }^{(b)}$ Time-span of the observation.

secular perspective acceleration, as well as instrumental drift. To reconstruct small systematic radial-velocity variations, so called nightly zero-point offsets, we use the measured RVs of all other stars with only small intrinsic RV variations from the respective observing nights (see Trifonov et al. 2018, 2020; Tal-Or et al. 2019, for details). Spectra without simultaneous FabryPérot drift measurements or a signal-to-noise ratio $(\mathrm{S} / \mathrm{N})$ lower than 10 are excluded during the process, which results in a total of $64 \mathrm{RV}$ measurements in the VIS and 66 in the NIR. The RVs show a median internal uncertainty of $2.1 \mathrm{~m} \mathrm{~s}^{-1}$ and a weighted rms (wrms) of $3.8 \mathrm{~m} \mathrm{~s}^{-1}$ in the VIS and 11.7 and $15.6 \mathrm{~m} \mathrm{~s}^{-1}$ in the NIR, respectively. The high scatter in the NIR channel corresponds to our expectation from the photon-noise limit considering the median measured $\mathrm{S} / \mathrm{N}$ of $\sim 63$ for the NIR observations (see Bauer et al. 2020, for a detailed analysis of the performance of CARMENES). Due to the low RV amplitude of the transiting planet candidate $\left(K \approx 2.2 \mathrm{~m} \mathrm{~s}^{-1}\right)$, we therefore used only the VIS data for this study.

IRD. In the course of the Subaru IRD TESS Intensive Follow-up Project (proposal S19A-069I), we observed GJ 3473 with the InfraRed Doppler spectrograph (IRD; Kotani et al. 2018), a near-infrared, adaptive-optics (AO) corrected, highresolution spectrograph $(0.97$ to $1.75 \mu \mathrm{m}, R \approx 70000)$ installed on the Subaru $8.2 \mathrm{~m}$ telescope. The integration time was set to $300-600$ s so that the extracted one-dimensional spectra have S/Ns of 50-70 per pixel at $1000 \mathrm{~nm}$. A total of 56 frames were acquired for GJ 3473 by IRD on 12 different nights between April 2019 and December 2019, all of which had simultaneous reference spectra of the laser frequency comb. The reduction of the raw data was performed with the IRAF echelle package (Tody 1993), including the wavelength calibration using thorium-argon hollow cathode lamps. For the RV analyses, wavelengths were re-calibrated more precisely based on the laser frequency comb spectra. RVs were measured using the forward modelling technique described by Hirano et al. (2020), in which the timevariable telluric absorptions and the instantaneous instrumental profile of the spectrograph were modelled and taken into account in the RV fits. The IRD RVs show a median internal uncertainty of $4.1 \mathrm{~m} \mathrm{~s}^{-1}$ and a wrms of $8.0 \mathrm{~m} \mathrm{~s}^{-1}$.

HARPS. GJ 3473 was also observed by the High Accuracy Radial velocity Planet Searcher (HARPS; Mayor et al. 2003) as part of the ESO programme 1102.C-0339(A). The spectrograph, installed at the ESO La Silla $3.6 \mathrm{~m}$ telescope, covers the spectral range from 0.378 to $0.691 \mu \mathrm{m}$ and has a resolution of $R=110000$. The 32 observations presented here were taken between May 2019 and March 2020. Their exposure times ranged between 30 and $40 \mathrm{~min}$. We use the reduced spectra from the HARPS Data Reduction Software (DRS; Lovis \& Pepe 2007) and compute their RVs following Astudillo-Defru et al. (2017a), which resulted in a lower rms scatter compared to the RVs retrieved with the SERVAL pipeline. They are calibrated for the barycentric motion, secular perspective acceleration, and instrumental drift. For the HARPS RVs, we obtain a median internal uncertainty of $3.4 \mathrm{~m} \mathrm{~s}^{-1}$ and a wrms of $4.8 \mathrm{~m} \mathrm{~s}^{-1}$.

\subsection{Ground-based transit follow-up}

The TFOP subgroup SG1 provides seeing-limited photometry follow-up observations of the TESS planet candidates in order to supplement the available photometry and to provide improved ephemerides for the targets. An overview of the observations, the instruments and the filters used is given in Table 1.

LCOGT. We used four transit observations of GJ 3473 from the Las Cumbres Observatory global telescope network (LCOGT; Brown et al. 2013). The observations were taken with the SINISTRO CCDs at the $1 \mathrm{~m}$ telescopes of the LCOGT, which have a pixel scale of $0.389 \mathrm{arcsec}_{\mathrm{pix}}^{-1}$ and a field of view (FOV) of $26 \operatorname{arcmin} \times 26$ arcmin each. The first transit was observed from the McDonald Observatory (McD) on 19 March 2019 in the $z_{s}$ filter, two transits were observed from the Cerro Tololo Interamerican Observatory (CTIO) on 21 and 27 February 2020 in $i_{p}$ filter and one transit in $z_{s}$ filter on 13 March 2020 from South African Astronomical Observatory (SAAO). We calibrated the images with the standard LCOGT Banzai pipeline (McCully et al. 2018) and extracted the light curves using AstroImage] (Collins et al. 2017).

MuSCAT. GJ 3473 was observed on 18 January 2020 by the Multi-color Simultaneous Camera for studying Atmospheres of Transiting planets (MuSCAT; Narita et al. 2015) mounted at the $1.88 \mathrm{~m}$ telescope at the Okayama Astro-Complex on Mt. Chikurinji, Japan. MuSCAT is a multi-colour instrument that performs imaging in the $g, r$ and $z_{s}$-filter bands at the same time. Each camera has a FOV of 6.1 arcmin $\times 6.1$ arcmin with a pixel scale of 0.358 arcsec pix $^{-1}$. Due to a large scatter in the $g$ band, we only use the $r$ and $z_{s}$ light curves here. The individual images are corrected for dark current and flat fields, and the light curves 
are generated using a custom pipeline that is described in Fukui et al. (2011).

MuSCAT2. We made use of two transit observations from MuSCAT2 (Narita et al. 2019). The instrument is mounted at the $1.52 \mathrm{~m}$ Telescopio Carlos Sánchez at the Observatorio del Teide, Spain. MuSCAT2 operates simultaneously in the $g, r$, $i$, and $z_{s}$ passbands and has a FOV of $7.4 \mathrm{arcmin} \times 7.4 \mathrm{arcmin}$ at $0.44 \operatorname{arcsec}_{\text {pix }}{ }^{-1}$ resolution. One transit was observed on 21 December 2019, from which we use the observations in the $i$ and $z_{s}$ bands. The other transit was observed on 2 January 2020, from which we use the observations in the $r, i$, and $z_{s}$ bands. Both transits were observed defocussed to optimise the quality of the photometry. The transit signal had too low $\mathrm{S} / \mathrm{N}$ in the $g$ band to be useful in the fitting, and the $r$ band observations were affected by systematics on the night of 21 December. The photometry was produced using a dedicated MuSCAT2 photometry pipeline (see Parviainen et al. 2019, for details) and the detrended light curves were created by a fit that aims to simultaneously choose the best target and comparison star apertures, model the systematics using a linear term, and include the transit using PyTransit (Parviainen 2015).

USAFA. We used the brand-new, recently commissioned $1 \mathrm{~m}$ USAFA Telescope, which is an optically-fast $f / 6$ RitcheyChrétien telescope with a wide field of view $0.9 \mathrm{deg}^{2}$ and an STA1600 CCD installed on the outskirts of Colorado Springs. We observed GJ 3473 on 04 March 2020. The USAFA data did not firmly detect the transit on target, but ruled out nearby eclipsing binaries in all other stars within the apertures of TESS, LCOGT, and MuSCAT/2 (Fig. 1).

\subsection{Photometric monitoring}

We used long-term photometric monitoring of GJ 3473 to search for periodic signals associated with the rotation period of the star.

TJO. We observed GJ 3473 with the $80 \mathrm{~cm}$ Joan Oró telescope (TJO) at Observatori Astronòmic del Montsec, Spain. The star was monitored between 31 January and 8 May 2020 for a total of 32 nights. Our observations were performed in the Johnson $R$ filter by using the main imaging camera LAIA, which has a $4 \mathrm{k} \times 4 \mathrm{k}$ back illuminated CCD with a pixel scale of 0.4 arcsec and a FOV of 30 arcmin. We calibrated each image for bias and dark current as well as applied flat field images using the ICAT pipeline (Colome \& Ribas 2006). Differential photometry was extracted with AstroImageJ using the aperture size and set of comparison stars that minimised the rms of the photometry. Low $\mathrm{S} / \mathrm{N}$ data due to high airmass or bad weather were removed. The data were binned to one measurement per hour. The median internal uncertainty is $2.7 \mathrm{ppt}$, while the rms is $9.4 \mathrm{ppt}$ around the mean. For the estimation of the stellar rotation period with a Gaussian process, we binned these data to one data point per night. This reduces short term variations caused by jitter and yields a median internal uncertainty of $2.9 \mathrm{ppt}$ and a rms of $7.4 \mathrm{ppt}$ around the mean.

MEarth. The all-sky transit survey MEarth consists of 16 robotic $40 \mathrm{~cm}$ telescopes with a FOV of $26 \mathrm{arcmin}^{2}$ located at two observatories in the southern and northern hemisphere (Berta et al. 2012). We use archival photometric monitoring data from the Mearth-North project DR8 ${ }^{7}$ covering the time span from 2008 to 2010 and 2011 to 2018. In total, we retrieved

\footnotetext{
7 https://www.cfa.harvard.edu/MEarth/DR8/
}

6220 observations of GJ 3473 from the archive. They were observed with telescopes 01 and 04 in the broad RG715 filter. For the photometric analysis of the host star, we use the individual nightly binned time series, which shows a median internal uncertainty of $2.6 \mathrm{ppt}$ and a rms of $8.7 \mathrm{ppt}$ around the mean.

\subsection{High-resolution imaging}

As part of the standard process for validating transiting exoplanets and to assess the possible contamination of bound or unbound companions on the derived planetary radii (Ciardi et al. 2015), high-resolution images of GJ 3473 were taken within the TFOP "High Resolution Imaging" SG3 subgroup.

Gemini/NIRI. Nine images of GJ 3473 in the $\mathrm{Br} \gamma$ narrow filter $\left(\lambda_{0}=2.1686 ; \Delta \lambda=0.0295 \mu \mathrm{m}\right)$ were taken with the NIRI instrument mounted at the $8.1 \mathrm{~m}$ Gemini North telescope (Hodapp et al. 2003) on 22 March 2019 as part of the Gemini programme GN-2019A-LP-101. The science frames had an exposure time of $3.5 \mathrm{~s}$ each and were dithered in a grid pattern with $\sim 100$ px spacing ( 2.2 arcsec). A sky background image was created by median combining the dithered images. The basic reduction included bad pixel interpolation, flatfield correction, sky background subtraction, and alignment and co-adding of the images.

Keck/NIRC2. The Keck Observatory observations were made with the NIRC2 instrument on the $10.0 \mathrm{~m}$ Keck II telescope behind the natural guide star AO system (Service et al. 2016, and references therein). The observations were taken on 25 March 2019 in the standard three-point dither pattern that is used with NIRC2 to avoid the left lower quadrant of the detector, which is typically noisier than the other three quadrants. The dither pattern step size was 3 arcsec and was repeated four times. The observations were also obtained in the narrow-band $\mathrm{Br} \gamma$ filter $\left(\lambda_{0}=2.1686 ; \Delta \lambda=0.0326 \mu \mathrm{m}\right)$ with an integration time of $20 \mathrm{~s}$ with one coadd per frame for a total of $300 \mathrm{~s}$ on target. The camera was in the narrow-angle mode with a full field of view of $\sim 10 \operatorname{arcsec}$ and a pixel scale of $0.099442 \operatorname{arcsec~pix}^{-1}$.

\section{Properties of GJ 3473}

The star GJ 3473 (G 50-16, Karmn J08023+033) was included in the TESS Input Catalogue as TIC 452866790 and declared a TESS Object of Interest (TOI) 488 after the transiting planet candidate was found. A summary of the stellar parameters is given in Table 2. The star was classified as an M4.0 V star by Hawley et al. (1996), but it was never subject to an in-depth analysis of its properties (e.g. Newton et al. 2014). To determine precise stellar parameters, we used the high-resolution spectra from the CARMENES follow-up observations. Following Passegger et al. $(2018,2019)$ and applying a measured upper limit of $v \sin i=2 \mathrm{~km} \mathrm{~s}^{-1}$, we calculated the effective temperature $T_{\text {eff }}, \log g$, and $[\mathrm{Fe} / \mathrm{H}]$ from a fit to a grid of PHOENIX stellar atmosphere models (Husser et al. 2013) using a $\chi^{2}$ method. The derived $T_{\text {eff }}$ matches the literature spectral type taking into account the uncertainties in both parameters (Alonso-Floriano et al. 2015; Passegger et al. 2018; Cifuentes et al. 2020). Next, we determined the bolometric luminosity, $L_{\star}$, by integrating the spectral energy distribution in 14 broad passbands from optical $B$ to W4 with the Gaia DR2 parallax (Gaia Collaboration 2018) as in Cifuentes et al. (2020). The radius, $R_{\star}$, was subsequently calculated using the Stephan-Boltzmann law. Lastly, the mass, $M_{\star}$, was derived from the Schweitzer et al. (2019) 
Table 2. Stellar parameters of GJ 3473.

\begin{tabular}{|c|c|c|}
\hline Parameter & Value & Ref. \\
\hline \multicolumn{3}{|c|}{ Name and identifiers } \\
\hline Name & GJ 3473 & Gli91 \\
\hline Alt. name & G 50-16 & Gic59 \\
\hline Karmn & $\mathrm{J} 08023+033$ & Cab16 \\
\hline TIC & 452866790 & Stas 19 \\
\hline TOI & 488 & TESS releases \\
\hline \multicolumn{3}{|c|}{ Coordinates and spectral type } \\
\hline$\alpha(\mathrm{J} 2000)$ & 080222.88 & Gaia DR2 \\
\hline$\delta(\mathrm{J} 2000)$ & +032019.7 & Gaia DR2 \\
\hline Sp. type & $\mathrm{M} 4.0 \mathrm{~V}$ & Haw96 \\
\hline$G[\mathrm{mag}]$ & $12.4650 \pm 0.0003$ & Gaia DR2 \\
\hline$T[\mathrm{mag}]$ & $11.1972 \pm 0.0073$ & Stas 19 \\
\hline$J[\mathrm{mag}]$ & $9.627 \pm 0.023$ & 2MASS \\
\hline \multicolumn{3}{|c|}{ Parallax and kinematics } \\
\hline$\mu_{\alpha} \cos \delta\left[\mathrm{mas} \mathrm{yr}^{-1}\right]$ & $-403.17 \pm 0.09$ & Gaia DR2 \\
\hline$\mu_{\delta}\left[\operatorname{mas~yr}^{-1}\right]$ & $-381.01 \pm 0.05$ & Gaia DR2 \\
\hline$\pi[$ mas $]$ & $36.52 \pm 0.05$ & Gaia DR2 \\
\hline$d[\mathrm{pc}]$ & $27.39 \pm 0.04$ & Gaia DR2 \\
\hline$\gamma\left[\mathrm{km} \mathrm{s}^{-1}\right]$ & $-1.101 \pm 0.011$ & This work \\
\hline$U\left[\mathrm{~km} \mathrm{~s}^{-1}\right]$ & $-3.11 \pm 0.05$ & This work \\
\hline$V\left[\mathrm{~km} \mathrm{~s}^{-1}\right]$ & $-27.66 \pm 0.06$ & This work \\
\hline$W\left[\mathrm{~km} \mathrm{~s}^{-1}\right]$ & $-66.44 \pm 0.07$ & This work \\
\hline \multicolumn{3}{|c|}{ Photospheric parameters } \\
\hline$T_{\text {eff }}[\mathrm{K}]$ & $3347 \pm 54$ & This work \\
\hline $\log g[\mathrm{dex}]$ & $4.81 \pm 0.06$ & This work \\
\hline$[\mathrm{Fe} / \mathrm{H}][\mathrm{dex}]$ & $+0.11 \pm 0.19$ & This work \\
\hline \multicolumn{3}{|c|}{ Physical parameters } \\
\hline$L_{\star}\left[L_{\odot}\right]$ & $0.01500 \pm 0.00019$ & This work \\
\hline$R_{\star}\left[R_{\odot}\right]$ & $0.364 \pm 0.012$ & This work \\
\hline$\hat{M}_{\star}\left[M_{\odot}\right]$ & $0.360 \pm 0.016$ & This work \\
\hline \multicolumn{3}{|c|}{ Activity parameters } \\
\hline $\mathrm{pEW}(\mathrm{H} \alpha)[\AA]$ & $+0.08 \pm 0.15$ & This work \\
\hline $\log R_{\mathrm{HK}}^{\prime}[\mathrm{dex}]$ & $-5.62 \pm 0.22$ & This work \\
\hline$v \sin i\left[\mathrm{~km} \mathrm{~s}^{-1}\right]$ & $<2$ & This work \\
\hline$P_{\text {rot }}[\mathrm{d}]$ & $168.3 \pm 4.2$ & This work \\
\hline
\end{tabular}

References. Gli91: Gliese \& Jahreiß (1991); Gic59: Giclas et al. (1959); Cab16: Caballero et al. (2016b); Stas19: Stassun et al. (2019); Gaia DR2: Gaia Collaboration (2018); Haw96: Hawley et al. (1996); 2MASS: Skrutskie et al. (2006).

empirical mass-radius relation derived from eclipsing binaries. The values determined in this way are consistent with the mass and radius determined from isochrones (Morton 2015). We updated the Galactocentric space velocities $U V W$ according to Cortés-Contreras (2016) by combining the Gaia DR2 parameters and the absolute velocity measured from the crosscorrelation function (CCF) of the spectra with a weighted binary mask (Lafarga et al. 2020). Using the space velocities and the BANYAN $\Sigma$ Bayesian classification tool, we found no indication that GJ 3473 is a member of any nearby young stellar association. Instead, it is most probably a field star located in the Galactic thin disk (Gagné et al. 2018).

GJ 3473 is listed in the Washington Double Star catalogue (Mason et al. 2001) as the primary of the binary system LDS 5160 (Luyten Double Star), with a date of first satisfactory observation in 1949. The secondary, at an angular separation of $49.29 \pm 0.09$ arcsec to the southeast of GJ $3473(\theta=222.8 \pm$ $14.1 \mathrm{deg}$ ), is LP 544-12 (GJ 3474, source \#3 in Fig. 1), a
$J=12.2$ mag M6 V star that shares the proper motion and parallax values of our planet-host star, but with a third of its mass (Luyten 1979; Reid et al. 1995; Newton et al. 2017). At the distance of GJ 3473, the angular separation between the two stars translates into a projected physical separation of $1349.9 \pm 3.1 \mathrm{au}$.

\section{Analysis and results}

\subsection{Transit search within the TESS light curve}

A transiting planet candidate around GJ 3473 was announced on 14 March 2020 via the TESS releases website ${ }^{8}$. The candidate passed all tests from the SPOC Data Validation Report (Twicken et al. 2018; Jenkins 2002; Li et al. 2019) and it is listed on the Exoplanet Follow-up Observing Program (ExoFOP) ${ }^{9}$ webpage as having a period of $1.1981 \mathrm{~d}$ and a transit depth of $1.051 \mathrm{ppt}$. We performed an independent transit search on the PDC-SAP light curve using the Transit-Least-Squares method (TLS; Hippke \& Heller 2019) ${ }^{10}$. We consider a signal to be significant if it reaches a signal detection efficiency (SDE; Alcock et al. 2000; Pope et al. 2016) of at least SDE $\geq 8$. The TLS shows a highly significant transit signal $(P \approx 1.1979 \mathrm{~d})$ with an SDE of $\sim 18.4$ and a transit depth of $1.071 \mathrm{ppt}$. After pre-whitening of the photometric data by fitting for this signal, a TLS of the residuals shows no remaining significant signals with $\mathrm{SDE} \geq 8$.

\subsection{Adaptive-optics imaging and limits of photometric contamination}

As part of our standard process for validating transiting exoplanets and to assess the possible contamination of bound or unbound companions on the derived planetary radii (Ciardi et al. 2015), we investigated the deep AO images from NIRC2 at Keck II and NIRI at Gemini North shown in Fig. 3. Both images were taken in Bry narrow filters. No companions are visible to a separation of 7.5 arcsec. The contrast curves are obtained by injecting fake sources of different brightness at different separations from the star and determining the radial $5 \sigma$ detection limit. The NIRC2 observations are sensitive up to a contrast of $\Delta m=8.3 \mathrm{mag}$ at a separation of $0.5 \mathrm{arcsec}$ to the star, but only span the region of the inner 3-4 arcsec. The area further out up to 6.0 arcsec is covered by the NIRI image, which reaches a contrast level of $\Delta m=7.9 \mathrm{mag}$. We therefore conclude, based on a combination of the contrast curves, a visual inspection of the AO images in Fig. 3, and the Baraffe et al. (2003) COND models for an approximate solar age, that GJ 3473 does not have a high-mass brown dwarf or more massive companion at $13-160 \mathrm{au}$. Furthermore, using additional 2MASS imaging and Gaia DR2 astro-photometry, we ruled out the presence of stellar companions of any mass at separations beyond $160 \mathrm{au}$ up to a few thousands au (excluding the known companion LP 544-12). Another indicator for binarity is the re-normalised a posteriori mean error of unit weight (RUWE), which quantifies the goodness-of-fit of the astrometric solution in the Gaia DR2 (Arenou et al. 2018; Lindegren et al. 2018). At approximate separations between 1.3 and $13 \mathrm{au}$, any hypothetical stellar companion would cause GJ 3473 to have a Gaia RUWE value larger than 1.41 (its actual value is 1.06; Cifuentes et al. 2020, and references therein) and an asymmetric point spread function in the NIRC2 and NIRI images. At separations closer

\footnotetext{
8 https://tess.mit.edu/toi-releases

9 https://exofop. ipac. caltech. edu/tess/target.php?id= 452866790

10 https://github. com/hippke/tls
} 


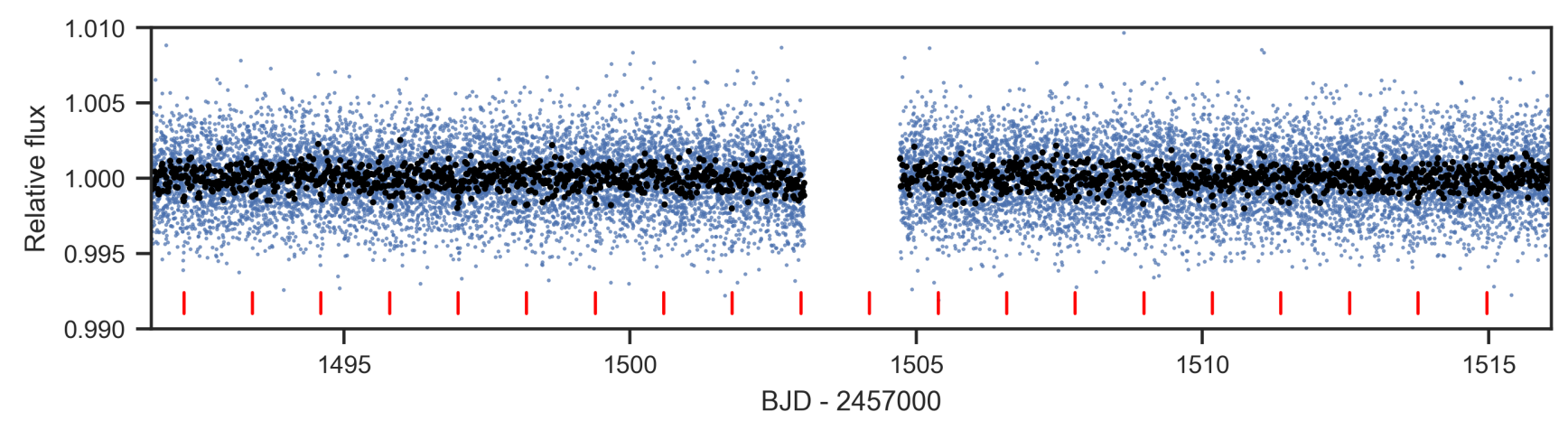

Fig. 2. TESS systematic-corrected PDC-SAP light curve. The blue dots are the measurements and the black dots are the data binned to 20 min. The transit times are marked by red ticks.
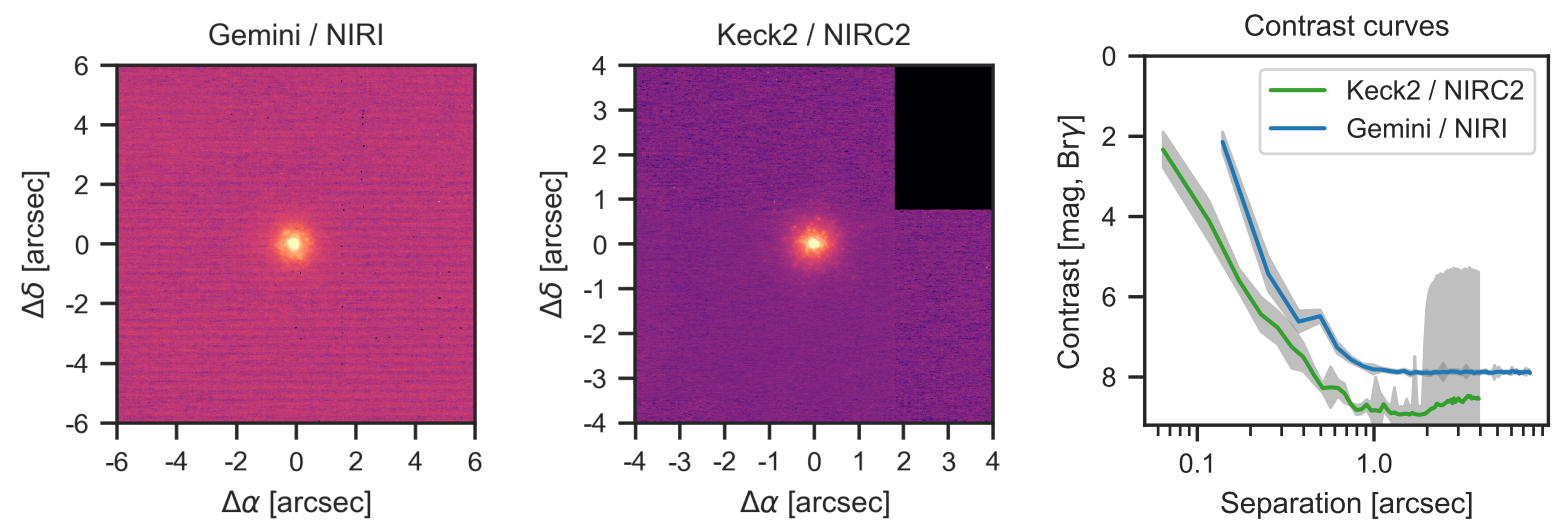

Fig. 3. AO images and contrast curves of the Keck II and Gemini North observations of GJ 3473. The grey shaded regions in the contrast curves are the uncertainty, which apparently rises dramatically for NIRC2 because of a dead quadrant.

than $1.3 \mathrm{au}$, we would see a double-peaked CCF or a longterm trend in the CARMENES RV data. We cannot exclude, however, the presence of substellar objects of a few Jupiter masses at wide separations (with orbital periods much longer than the RV coverage) or unfavourably aligned objects at close separations.

Additionally, we assessed the photometric contamination of the TESS light curves using Eq. (6) from Espinoza et al. (2019b). From the AO images, we obtained upper limits from 5 to 8 mag in contrast for the inner area from 0.15 to $7.5 \mathrm{arcsec}$, which correspond to contamination below $1 \%$. For the nearby Gaia sources apparent in Fig. 1, we make use of the fact that the TESS and Gaia $G_{\mathrm{RP}}$-band filter are very similar. We find that for the brightest nearby source (\# 3 in Fig. 1), which is its binary companion LP 544-12, the dilution factor would be 0.96 . However, given the separation of 48.9 arcsec to GJ 3473, this is negligible and, thus, we assume for our modelling that there are no contaminating sources nearby.

\subsection{Transits only modelling}

In order to refine the orbital period of the transiting planet candidate that was determined from the TLS analysis and to evaluate whether the individual follow-up observations show adequate transit detections, we first investigated the photometric observations separately from the RV measurements.

For all modelling tasks in this work, we used juliet ${ }^{11}$ (Espinoza et al. 2019b), a fitting tool that uses nested sampling to efficiently evaluate the parameter space of a given

${ }_{11}$ https://juliet.readthedocs.io/en/latest/ prior volume and to allow for model comparison based on Bayesian evidences. Here, juliet combines publicly available packages for RVs and transits, namely, radvel ${ }^{12}$ (Fulton et al. 2018) and batman ${ }^{13}$ (Kreidberg 2015). It allows us to choose among a range of different nested sampling algorithms for the fitting. We opted for dynesty ${ }^{14}$ (Speagle 2020) because of its simple usage with regard to multi-processing. Additionally, juliet provides the implementation of Gaussian processes in the models using either george ${ }^{15}$ (Ambikasaran et al. 2015) or celerite $^{16}$ (Foreman-Mackey et al. 2017).

As a first step, we modelled all of the 15 available ground based follow-up observations of transit events obtained by the TFOP SG $1^{17}$ separately with the TESS light curve while fitting for the transit centre of each transit (see Eastman et al. 2019, and the documentation of juliet for details of the implementation). In doing so, we re-parametrised the scaled semi-major axis to the stellar density, $\rho_{*}$. In this manner we can make use of the derived stellar parameters to obtain a density estimation as a fit prior. Furthermore, we implement the parameter transformation suggested by Espinoza (2018) and fit for the parameters, $r_{1}$ and $r_{2}$, instead of the planet-to-star radius ratio, $p$, and the impact parameter, $b$. A quadratic limb-darkening model is used for the TESS data (Espinoza \& Jordán 2015), which is parametrised by the $q_{1}$ and $q_{2}$ parameters (Kipping 2013), while a linear model is used

\footnotetext{
12 https://radvel.readthedocs.io/en/latest

13 https://www. cfa.harvard.edu/ 1kreidberg/batman/

14 https://github.com/joshspeagle/dynesty

15 https://george.readthedocs.io/en/latest/

16 https://celerite. readthedocs.io/en/stable

17 As of 13 March 2020.
} 


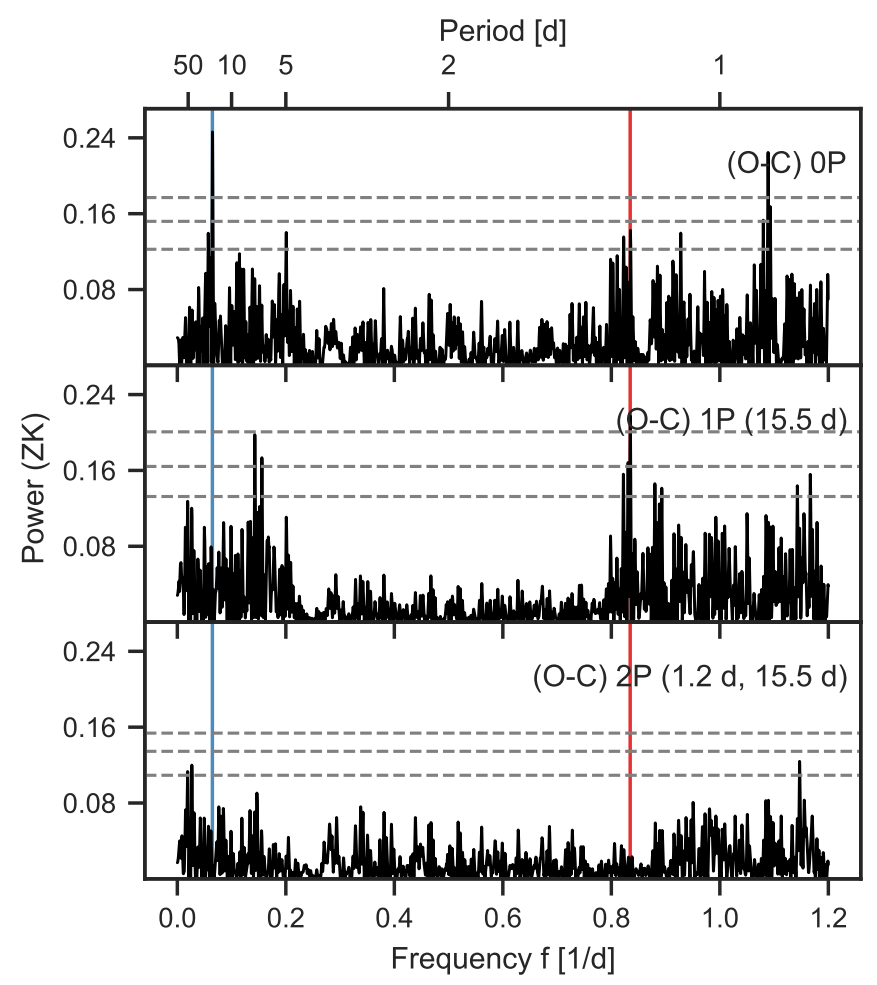

Fig. 4. GLS periodograms of the RV measurements. Vertical lines mark the transiting planet (b, solid red) and the new RV planet (c, solid blue) The horizontal dashed grey lines show the false alarm probability (FAP) of 10,1 , and $0.1 \%$ determined from 10000 random realisations of the measurements.

for the ground-based follow-up observations. We used a linear term to detrend the LCOGT and MuSCAT light curves with airmass, while the MuSCAT2 light curves were pre-detrended (see Sect. 2.3). Based on the results from Sect. 4.2, we fix the dilution factor to one for all instruments, but consider free individual instrumental offsets. Also instrumental jitter terms are taken into account and added in quadrature to the nominal instrumental errorbar.

By carrying out this pre-analysis, we were able to disregard observations that show no, or only marginal transits, or seem to be only apparent transits with transit centres far from a linear ephemeris. The final dataset, which is presented in Sect. 2 and which we use for the analysis in this work, includes 7 transit events with 11 observations of firm transit detections (cf. Table 1).

In the next step, we combine these observations and then fit for a common period and time of transit centre that serve as a basis for the joint analysis. In doing so, we determine $P=1.1980034_{-0.0000023}^{+0.0000022} \mathrm{~d}$ and $t_{0}=2458492.20410_{-0.00050}^{+0.00052}$.

\subsection{RV only modelling}

We approach the analysis of the RVs with a signal search in the data, proceeding as if we do not know of the transiting planet a priori. In Fig. 4, the generalised Lomb-Scargle periodograms (GLS; Zechmeister \& Kürster 2009) of the residuals from different fits of increasing complexity to the combined RVs from CARMENES, IRD, and HARPS are shown. We normalised the periodograms using the parametrisation of Zechmeister \& Kürster (2009, ZK). For all fits, we used Gaussian distributed priors for the signal of the transiting planet candidate based on the results from Sect. 4.3 and a narrow uniform range around the peak of the second signal. Instrumental offsets and jitter are treated separately for each dataset. For comparison, we list the Bayesian evidences of the fits in Table 4.

The first panel in Fig. 4 shows the periodogram of the residuals after fitting a flat model that only includes offsets and instrumental jitter to the CARMENES, IRD, and HARPS measurements. The strongest signal apparent in the RV data is a period at $15.5 \mathrm{~d}$. After subtracting this periodicity with a circular Keplerian fit, the residual periodogram shows a significant signal (FAP $<1 \%$ ), coincident with the period of the transiting planet at $P \approx 1.198 \mathrm{~d}$ (see the middle panel of Fig. 4). The FAP for a signal to occur especially at this frequency can be evaluated using the method by Baluev (2008) and the power of the signal in a Lomb-Scargle periodogram. By this means, we find a spectral FAP $\approx 0.003 \%$. We verify this using a bootstrap method of $1 \times 10^{6}$ random realisations over a decreasing frequency range centred on the period in question, which yields FAP $\approx 0.002 \%$. This is in agreement with the Baluev method and we therefore assume a FAP of 0.002 to $0.003 \%$ for the signal to occur at the expected period. Furthermore, the phase of this signal matches the phase of the planet candidate from TESS and we thus report a highly significant detection of the transiting planet candidate in the RV. The two other signals of significant power at periods of 6.41 and $7.00 \mathrm{~d}$ are aliases of the former signal of the transiting planet due to the approximately daily sampling. This is reflected by the fact that they disappear when the $15.5 \mathrm{~d}$ signal is fitted together with the period of the transiting planet at $P \approx 1.198 \mathrm{~d}$ (see the bottom panel of Fig. 4). Given that RV data provide more information on the eccentricity of an orbit, we performed an analogous analysis using eccentric orbits. We find that the difference between a circular and eccentric orbit is indistinguishable $(\Delta \ln \mathcal{Z}=-0.45)$ and, therefore, we use the results for the simpler circular model fits. The residuals of this fit comprising two circular Keplerian signals do not show any further periodicities with FAPs above our significance criterion of $1 \%$.

\subsection{Joint modelling}

For the final retrieval of the most precise parameters we perform a joint fit of the TESS observations, the ground-based transit follow-ups, and the combined RV data. The model includes two circular planets: firstly, the transiting planet that is detected in the photometry and RV data and; secondly, a non-transiting planet that is only apparent in the RV data. The model of the joint fit comprises 58 free parameters, which turns the fit into a high-dimensional problem. A fit with uninformed priors would therefore be very costly. Hence, we make use of the findings from the photometry-only analysis in Sect. 4.3 and the RV-only analysis in Sect. 4.4 - that is, we implement Gaussian distributed priors for the planetary parameters, as, for example, in Brahm et al. (2019), Espinoza et al. (2019a), Kossakowski et al. (2019), Luque et al. (2019); or Bluhm et al. (2020). Since we use uninformed priors for the planetary parameters for the transit and RV-only fits, nested sampling warrants an efficient exploration of the possible parameter space fitting the individual datasets. Planetary parameters specific to a given data type, such as the planetary semi-amplitude in RV data or planet-to-star radius (and others) in transit data, would not change significantly in a joint fit as they are independent from the other data to first order. Generally, this also holds true for the shared parameters since they are mostly driven by either one or the other method. Likewise, using the posterior results from the transit-only and RV-only fits as a prior knowledge for the joint fit is therefore justified given 

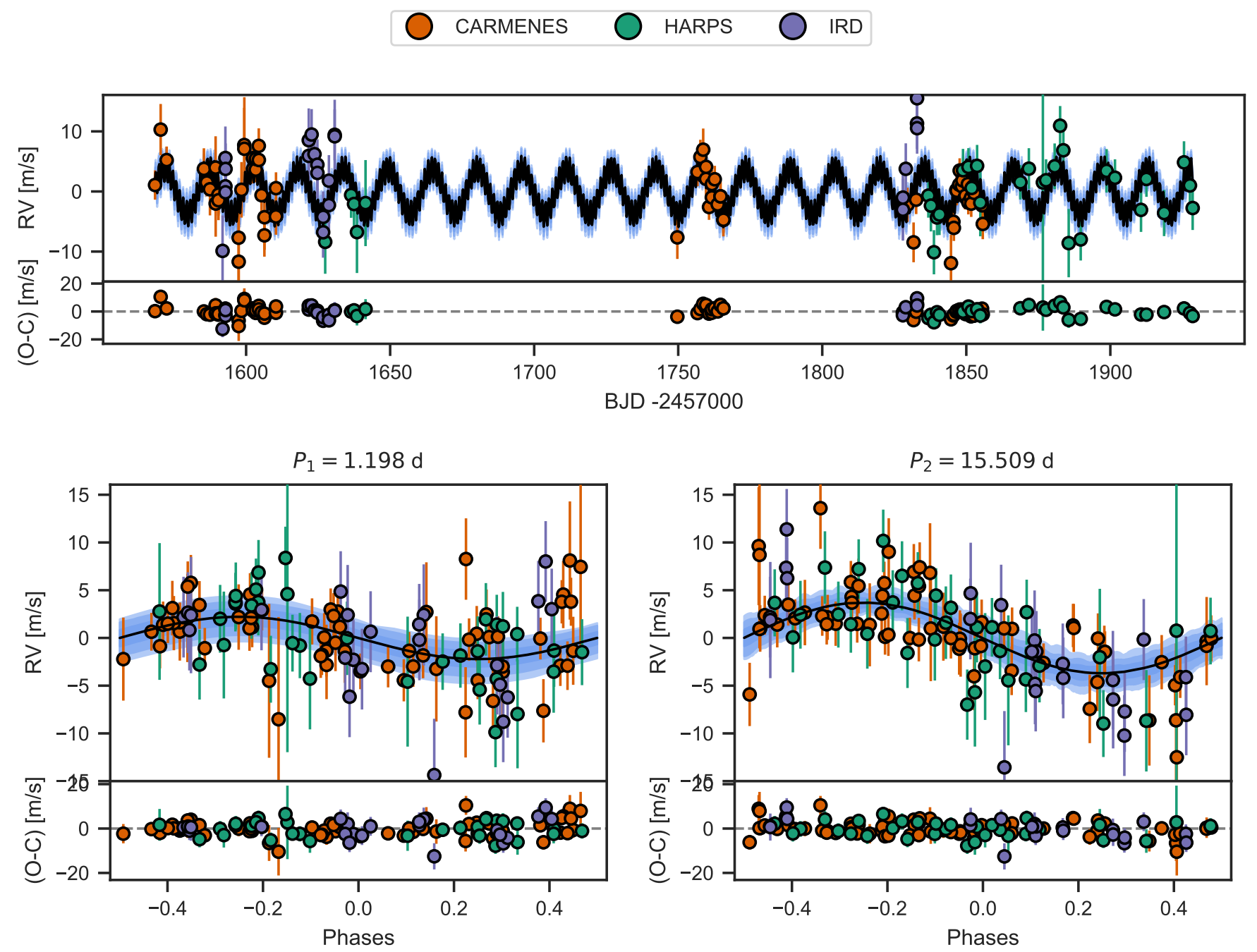

Fig. 5. Results from the joint fit for the RV data of CARMENES, IRD, and HARPS. The top part of each panel shows the measurements as coloured circles - errorbars include the instrumental jitters added in quadrature - and the median of the best-fit juliet model as the black curve. The grey shaded areas mark the 68,95 , and $99 \%$ credibility intervals. To avoid overcrowding of the figure, we binned the IRD data, which were taken with a high cadence, to chunks of $30 \mathrm{~min}$ each. In the lower part, the residuals after the model is subtracted (O-C) are shown. Top panel: RVs over time. Bottom panels: RVs phase-folded to the periods of the transiting planet (left) and the new RV planet (right).

that the chosen prior volume for the joint fit does not restrict the posterior volume from the individual fits. Following this, the width of the priors that we choose for the planetary parameters of the joint fit are three times the standard deviation of the posterior results from the individual best fits. It limits the computational cost, but still allows the nested sampling algorithm to freely explore the parameter space since the Gaussian distribution has no strict borders. In the end, the posterior distribution of our joint fit is much narrower than that of the input priors, which shows that the input priors were conservatively chosen to map the relevant parameter space and did not reject crucial possible solutions. Descriptions and justifications of the adopted instrumental parameters and priors can be found in the respective subsections, Sects. 4.3 and 4.4. A summary of the used priors is given in Table B.1.

In Figs. 5 and 6, we show the final models of the joint fit based on the posterior of the sampling. The median posteriors of the planetary parameters are shown in Table 3 and the full list of the posteriors of the instrumental parameters is given in Table C.1.

\subsection{Stellar activity}

We investigated a set of activity indicators derived from the CARMENES spectra to search for signals of stellar activity that would interfere with the transiting planet candidate or provide information on the origin of the second periodicity that is visible in the RV data (see Sect. 4.4). In Fig. 7, the GLS periodograms of 13 selected activity indicators, as well as our applied nightly zero-point offsets, are shown. The chromatic index (CRX) and the differential line width (dLW) are products of the SERVAL reduction pipeline (Zechmeister et al. 2018). From the CCF (see Sect. 3), the full-width at half-maximum (FWHM), the contrast, and the bisector span are determined (Lafarga et al. 2020). The pseudo-equivalent width after subtraction of an inactive template spectrum ( $\mathrm{pEW}^{\prime}$ ) of the chromospheric $\mathrm{H} \alpha$, Ca II IRT ( $\mathrm{a}, \mathrm{b}$ and c), He I $\lambda 10833 \AA$ and $\mathrm{He}_{\mathrm{I}} \mathrm{D}_{3}$ lines, and the photospheric $\mathrm{TiO} \lambda 7050 \AA$ and $\mathrm{TiO} \lambda 8430 \AA$ indices are calculated following Schöfer et al. (2019).

A measured median pEW of the $\mathrm{H} \alpha$ line of $+0.08 \pm 0.15 \AA$ indicates that GJ 3473 is a rather inactive star (Jeffers et al. 2018). We find a significant, although moderate, correlation between 

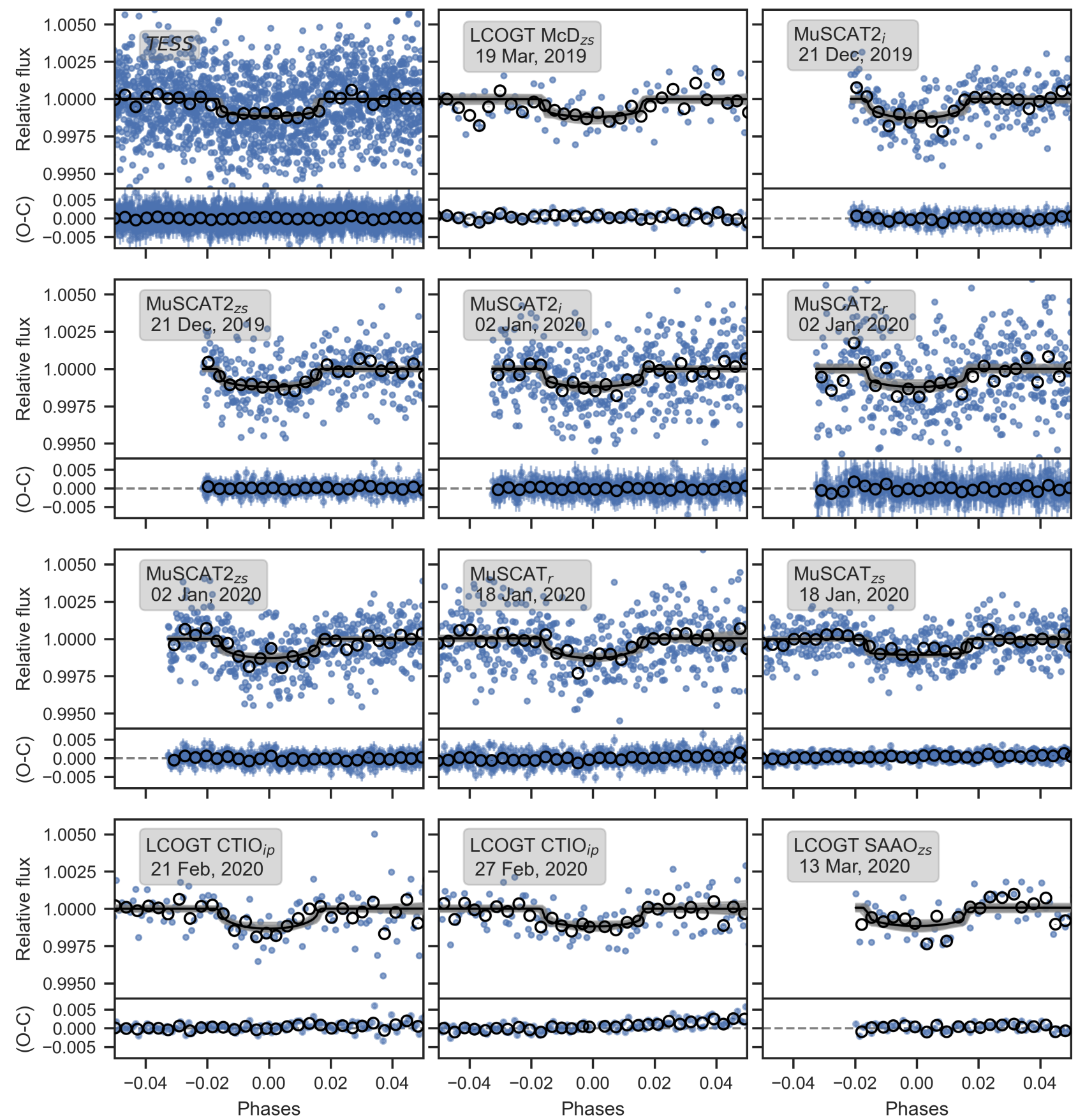

Fig. 6. Results from the joint fit for the transit observations. In the top part of each panel, the black curve presents the best-fit juliet model together with the 68,95 , and $99 \%$ credibility intervals displayed by the grey shaded regions. The observations of the respective instruments are phase-folded to the period of the transiting planet. For the fit, the individual data points (blue) are used, but the binned data are also shown for clarity (white circles). Error bars of the individual measurements with the instrumental jitter terms added in quadrature are only displayed in the bottom part of the panels, which show the residuals after subtracting the model $(\mathrm{O}-\mathrm{C})$. The names of the instruments and the dates of the observations are denoted in the grey boxes in the upper left corner of each panel.

$\mathrm{RV}$ and the CRX and Na I D activity indices, however, the GLS periodograms from the extensive set of activity indicators do not show any power at the frequencies of the transiting planet candidate or the $15.5 \mathrm{~d}$ signal. The $\mathrm{dLW}, \mathrm{CCF}$ contrast, TiO $\lambda 7050 \AA$, and He I $\lambda 10833 \AA$ show a forest of signals with $1 \%<$ FAP $<10 \%$ in the range of approximately 30 to $100 \mathrm{~d}$. This is consistent with a lower limit of the stellar rotation period to be longer than $\sim 9 \mathrm{~d}$ as determined from $v \sin i<2 \mathrm{~km} \mathrm{~s}^{-1}$, however, there is no common periodicity or conclusive pattern, which would hint at the rotation period of the star. The most significant signal, which is apparent in the He I $\lambda 10833 \AA$ indicator, has a period of around $100 \mathrm{~d}$. From the HARPS spectra we derive $\log R_{\mathrm{HK}}^{\prime}=-5.62 \pm 0.22$, which is equivalent to a stellar rotation period of $109(37)$ d following the $R_{\mathrm{HK}}^{\prime}$ vs. $P_{\text {rot }}$ relationship of Astudillo-Defru et al. (2017b). We also investigated GLS periodograms of the HARPS activity indicators derived by the DRS pipeline, but we do not find any significant periodicity and, therefore, we do not present them here. 
Table 3. Posterior parameters of the joint fit of the transit and RV data.

\begin{tabular}{|c|c|c|}
\hline Parameter & Posterior $^{(a)}$ & Units \\
\hline \multicolumn{3}{|c|}{ Stellar parameters } \\
\hline$\rho_{\star}$ & $10.93_{-0.69}^{+0.66}$ & $\mathrm{~g} \mathrm{~cm}^{-3}$ \\
\hline & Planetary parameters & \\
\hline$P_{\mathrm{b}}$ & $1.1980035_{-0.0000019}^{+0.0000018}$ & d \\
\hline$t_{0, \mathrm{~b}}$ & $2458492.20408_{-0.00042}^{+0.00043}$ & $\mathrm{~d}$ \\
\hline$r_{1, \mathrm{~b}}$ & $0.557_{-0.049}^{+0.044}$ & $\ldots$ \\
\hline$r_{2, \mathrm{~b}}$ & $0.03184_{-0.00067}^{+0.00069}$ & $\ldots$ \\
\hline$K_{\mathrm{b}}$ & $2.21_{-0.35}^{+0.35}$ & $\mathrm{~m} \mathrm{~s}^{-1}$ \\
\hline$\sqrt{e_{\mathrm{b}}} \sin \omega_{b}$ & 0 (fixed) & $\ldots$ \\
\hline$\sqrt{e_{\mathrm{b}}} \cos \omega_{\mathrm{b}}$ & 0 (fixed) & $\ldots$ \\
\hline$P_{\mathrm{c}}$ & $15.509_{-0.033}^{+0.033}$ & $\mathrm{~d}$ \\
\hline$t_{0, \mathrm{c}}$ & $2458575.62_{-0.43}^{+0.42}$ & d \\
\hline$K_{\mathrm{c}}$ & $3.75_{-0.42}^{+0.45}$ & $\mathrm{~m} \mathrm{~s}^{-1}$ \\
\hline$\sqrt{e_{\mathrm{c}}} \sin \omega_{\mathrm{c}}$ & 0 (fixed) & $\ldots$ \\
\hline$\sqrt{e_{\mathrm{c}}} \cos \omega_{\mathrm{c}}$ & 0 (fixed) & $\cdots$ \\
\hline
\end{tabular}

Notes. ${ }^{(a)}$ Error bars denote the $68 \%$ posterior credibility intervals. The posteriors of the instrumental parameters are continued in Table C.1.

\subsection{Photometric stellar rotational period}

We combined the $R$-band TJO data collected between January and May 2020 and the RG715-band MEarth data taken between 2008 and 2018 to determine a stellar rotation period. A marginalised likelihood periodogram (MLP; Feng et al. 2017) analysis of the combined data, where we fit for jitter and offsets between the datasets, indicated a preliminary periodicity of $160 \mathrm{~d}$. The MLP uses sinusoidal functions to model possible significant signals. However, stellar activity tends to be quasi-periodic and can also deviate significantly from a simple sinusoidal. Thus, we used a Gaussian process (GP) to fit the photometry in a second approach.

We used juliet and select the quasi-periodic kernel by george for the modelling of the photometric data:

$k_{i, j}(\tau)=\sigma_{\mathrm{GP}}^{2} \exp \left(-\alpha \tau^{2}-\Gamma \sin ^{2}\left(\pi \tau / P_{\text {rot }}\right)\right)$,

where $\sigma_{\mathrm{GP}}$ is the amplitude of the GP component given in ppt (or $\mathrm{m} \mathrm{s}^{-1}$ when applied to RV data), $\Gamma$ is the amplitude of GP sine-squared component, $\alpha$ is the inverse length-scale of the GP exponential component given in $\mathrm{d}^{-2}, P_{\text {rot }}$ is the period of the GP quasi-periodic component given in days, and $\tau=\left|t_{i}-t_{j}\right|$ is the temporal distance between two measurements. To perform a blind search for quasi-periodic signals with the GP model, we put in uninformed priors for $\sigma_{\mathrm{GP}}, \Gamma_{i}$, and $\alpha$, but take a uniform range from 2 to $200 \mathrm{~d}$ for $P_{\text {rot }}$.

In doing so, the data of each instrument are averaged into nightly bins because of the large dataset and the computationally expensive log-likelihood evaluation of the used kernel. A daily sampling of the photometry is reasonable since we are searching for signals with periods of at least multiple days (see Sect. 4.6). Furthermore, binning reduces short-term variations due to jitter and decreases the uncertainties of the data points. For the GP model, we consider that each dataset can have different solutions for the amplitude parameters, $\sigma_{\mathrm{GP}}$ and $\Gamma$. This accounts for the possibility that the stellar activity depends on

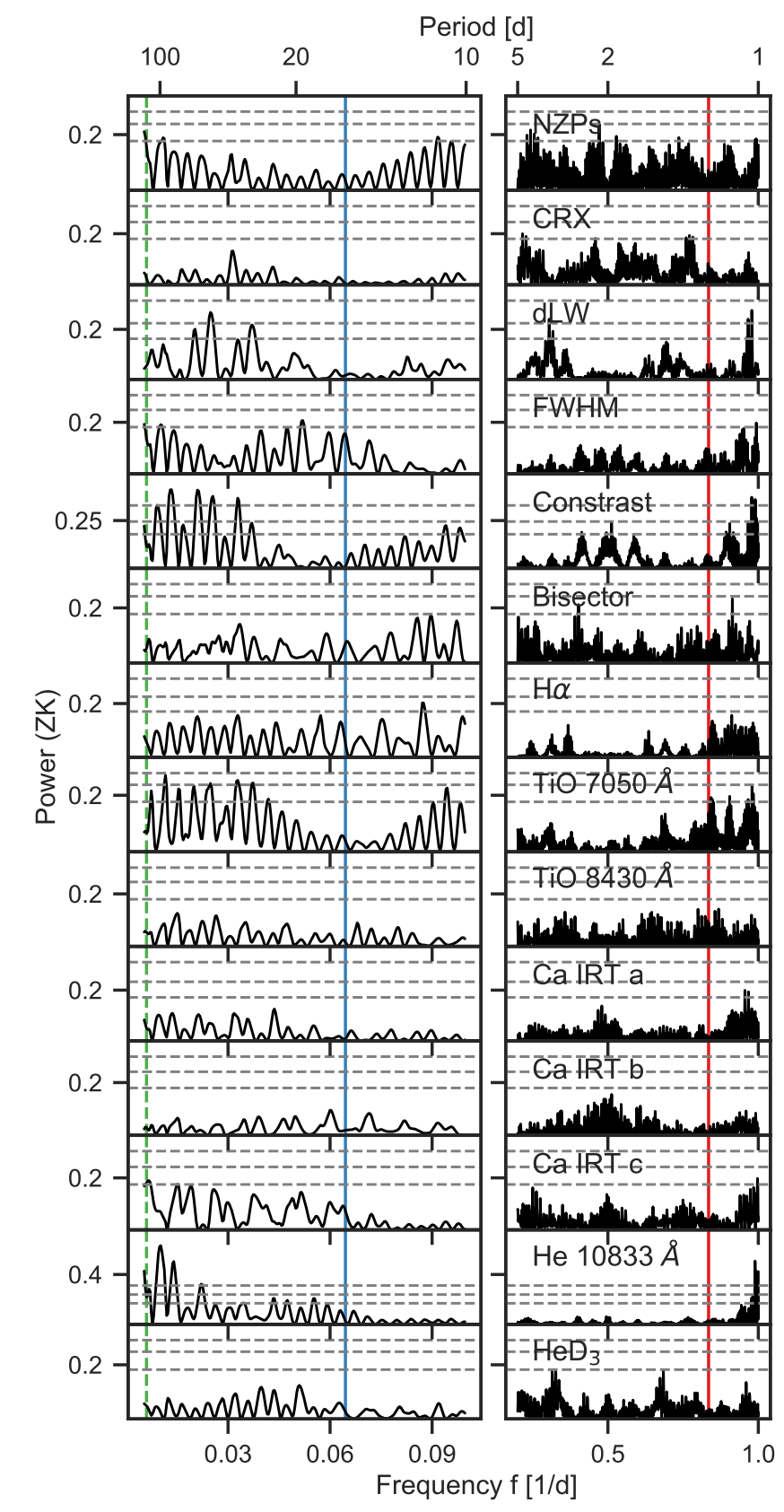

Fig. 7. GLS periodograms of a number of activity indicators based on spectroscopic data obtained by CARMENES, split into two frequency ranges. The vertical lines mark the frequencies of the transiting planet candidate (red solid), the 15.5 d periodicity visible in the RV (blue solid; see Sect. 4.4), and the determined photometric rotation period (dashed green; see Sect. 4.7). The horizontal grey lines show the false alarm probability (FAP) of 10,1 , and $0.1 \%$ determined from 10000 random realisations of the measurements.

wavelength and might impact each instrument differently. However, the timescale parameters, such as the rotational period, $P_{\text {rot }}$, and the exponential decay of the signal $\alpha$, for example, due to spot-life time, should not depend on the instrument. For the latter two parameters, we therefore allow only for global solutions of the GP model. We also model the flux offset between the photometric datasets, as well as an extra jitter component, which is added in quadrature to the diagonal of the resulting covariance matrix. Our GP fit, using unconstrained priors (Table B.2), results in only one specific region within the prior volume that 

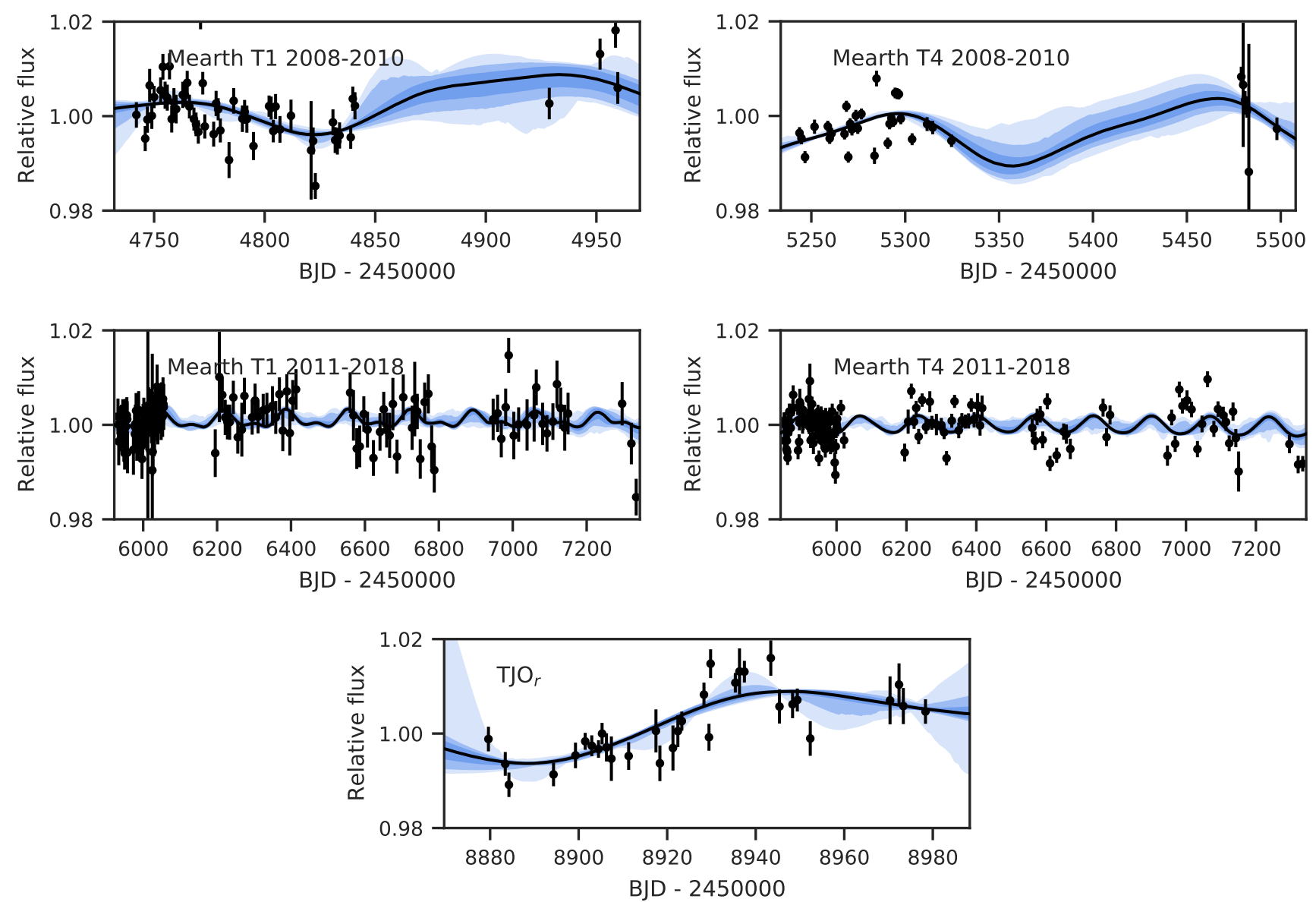

Fig. 8. Gaussian process fits to the photometric monitoring data of GJ 3473. From top to bottom: MEarth T1 2008-2010, MEarth T4 2008-2010, MEarth T1 2011-2018, MEarth T4 2011-2018, and TJO. The black line shows the median GP model extracted for each instrument and the blue shades denote the 68,95 and $99 \%$ confidence intervals.

has a high density of posterior samples with high likelihood. We show the nightly binned photometric data and the GP fit with its uncertainties in Fig. 8.

From the posterior solutions we derive a photometric rotation period, $P_{\text {rot,phot }}=168.3_{-3.1}^{+4.2} \mathrm{~d}$ for GJ 3473, which is consistent with the result from the MLP analysis and, within $2 \sigma$, with the expected period from $\log R_{\mathrm{HK}}^{\prime}$. Both estimates show that GJ 3473 is a slow rotator, which should not exhibit strong signals related to activity. This is also in agreement with the spectroscopic activity indicators, which do not exhibit a predominant periodicity and no $\mathrm{H} \alpha$ activity.

\subsection{Investigation of the $15.5 d$ signal}

The $15.5 \mathrm{~d}$ signal seems to be unrelated to stellar activity or the stellar rotation period. Following Fig. 5, the signal looks stable for the entire period of observations and shows no significant deviations from a circular Keplerian motion. However, we thoroughly examined the signal in order to asses its nature and to test whether we can attribute it unambigously to a planetary origin.

We used juliet to perform a model comparison based on the Bayesian evidence of different models, applied to the RV data only, in order to check whether the $15.5 \mathrm{~d}$ signal is indeed best fit with a Keplerian model. The log-evidences of the results are shown in Table 4. As outlined by Trotta (2008), we consider a difference of $\Delta \ln \mathcal{Z}>2$ as weak evidence that one of the models is preferred over the others and $\Delta \ln \mathcal{Z}>5$ that a model is
Table 4. Bayesian log-evidences for the different models used to fit the RVs.

\begin{tabular}{lccc}
\hline \hline Model & $\begin{array}{c}\text { Periods } \\
{[\mathrm{d}]}\end{array}$ & $\ln \mathcal{Z}$ & $\Delta \ln \mathcal{Z}$ \\
\hline 0 Planets & $\ldots$ & $-474.6 \pm 0.2$ & 0 \\
1 Planet & 1.20 & $-468.1 \pm 0.2$ & 6.5 \\
1 Planet & 15.5 & $-456.7 \pm 0.3$ & 17.9 \\
1 Planet + GP & 1.20 & $-444.9 \pm 0.3$ & 29.7 \\
2 Planets & $15.5,1.20$ & $-442.7 \pm 0.3$ & 31.9 \\
\hline
\end{tabular}

Notes. ${ }^{(a)}$ Rounded to three digits.

significantly favoured. We use Gaussian distributed priors based on the posterior solutions from Sect. 4.3 to account for the transiting planet candidate, and uniform priors for instrumental offsets and jitter. However, we adopted two approaches to include the $15.5 \mathrm{~d}$ signal in the modelling: on the one hand, a simple two-planet model is fitted to the data and on the other hand, we implement a quasi-periodic GP (see Eq. (1) in Sect. 4.7) to test the possibility that the second signal does not have a Keplerian nature and is only of a quasi-periodic origin, for example, due to stellar activity. We find a difference of $(\Delta \ln \mathcal{Z}=2.2)$ in favour of the two-planet model compared to the model, including a quasiperiodic component for the $15.5 \mathrm{~d}$ signal. This offers only weak 

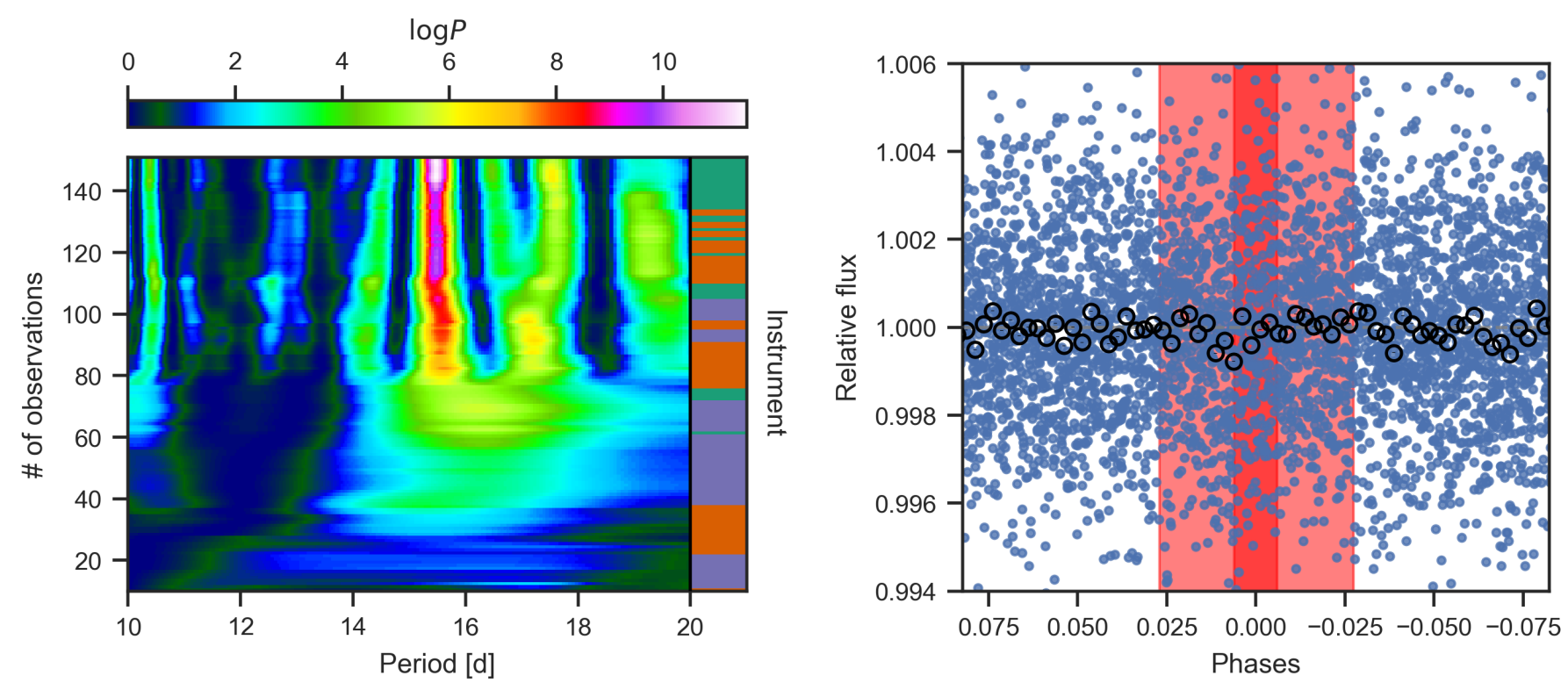

Fig. 9. Investigation of the $15.5 \mathrm{~d}$ signal Left: stacked BGLS periodogram of the residuals after fitting for the transiting planet. The colourbar on the right side indicates the instrument of the corresponding data point (orange: CARMENES, purple: IRD, green: HARPS). Right: TESS light curve phase-folded to the period and time of transit centre of GJ $3473 \mathrm{c}$ as determined from the RVs. The saturated red shaded region indicates the expected transit, while the light red shaded region denotes the $68 \%$ credibility interval of the time of transit centre.

evidence, confirming, nonetheless, that the signal is legitimately fitted by a Keplerian model.

Another way to test the coherence of a signal for a given dataset is the use of the so-called stacked Bayesian generalised Lomb-Scargle periodogram (s-BGLS; Mortier et al. 2015). The diagram in the left panel of Fig. 9 shows the probability of the $15.5 \mathrm{~d}$ signal normalised to the minimum of the considered frequency range (Mortier \& Collier Cameron 2017) for an increasing number of observations. The period of the signal is uncertain when only a few observations are included, but starting with more than 80 observations, a signal of consistently rising probability is detected at the period in question. This indicates that the signal is stable in phase and amplitude over the whole observational period of a $360 \mathrm{~d}$ time baseline, as is likely for a planetary signal. A colour-coded bar on the right side of the diagram specifies which of the instruments the considered data points originate from. As there are no variations of the signal caused by chunks of data from one specific instrument, we can also conclude that the signal is consistent between the different instruments.

Even though there are no obvious signs of more than one transiting planet in the TESS light curve (see Sect. 4.1), we searched for transits of the $15.5 \mathrm{~d}$ signal based on its parameters derived from the RV observations. Since the period of the planet is larger than half of the time span of the TESS data, which comprise only one sector, a potential transit is likely to occur only once in the data. The right panel in Fig. 9 shows the TESS data phase-folded to the expected time of transit centre. No obvious transit signals are visible. However, to quantify whether in fact there is no transit signal, we ran two more juliet fits on the TESS data using Gaussian distributed priors based on the posterior of the planetary parameters in Table 3 . The model considering only the transiting planet is favoured by $\Delta \ln \mathcal{Z} \approx 3.6$ over the model that treats the second periodicity as a transiting planet. Thus, we conclude that no significant transiting signal is associated with the $15.5 \mathrm{~d}$ periodicity.

\section{Discussion}

\subsection{GJ $3473 b$}

Our derived mass and radius confirm the planetary nature of the transiting planet candidate detected by TESS. GJ $3473 \mathrm{~b}$ has a mass of $1.86_{-0.30}^{+0.30} M_{\oplus}$ and a radius of $1.264_{-0.049}^{+0.050} R_{\oplus}$, which correspond to a density of $5.03_{-0.93}^{+1.07} \mathrm{~g} \mathrm{~cm}^{-3}$ and, thus, fits in the regime of Earth-sized planets with a density consistent with a composition dominated by $\mathrm{MgSiO}_{3}$ (see Fig. 10). A summary of the derived physical parameters of the planet can be found in Table 5 .

With an insolation flux of $59.4 \pm 5 S_{\oplus}$, GJ $3473 \mathrm{~b}$ is one of the hottest transiting Earth-mass planets with a dynamical mass measurement that has been detected so far (see Fig. 11). Its equilibrium temperature corresponds to $773 \pm 16 \mathrm{~K}$, assuming a zero Bond albedo. If the planet had an atmosphere, thermochemical equilibrium calculations predict water and methane to be the dominant opacity sources in the near/mid infrared (NIR/MIR) of the transmission spectrum of GJ $3473 \mathrm{~b}$, assuming a cloud-free solar-abundance scenario (e.g. Madhusudhan 2012; Mollière et al. 2015; Molaverdikhani et al. 2019a); see the red line in Fig. 12. In this scenario, the main transmission spectral features in the optical are expected to be alkali ( $\mathrm{Na}$ and $\mathrm{K}$ ), although their expected strength depends on a number of parameters such as the planetary atmospheric metallicity. The emission spectrum is heavily muted by water and methane absorption, causing very low relative flux at wavelengths shorter than $\sim 3 \mu \mathrm{m}$; see the blue line in Fig. 12. The dominant spectral features of a cloudy atmosphere in the optical and NIR are expected to be similar to those of a cloud-free atmosphere, although with lower amplitudes and less pronounced methane features (Molaverdikhani et al. 2020).

In addition, disequilibrium processes could change the composition and thermal structure of the planetary atmosphere. Depending on the exact temperature structure and methane abundance profile, vertical mixing could lead to methane quenching (e.g. Molaverdikhani et al. 2019b). Hydrocarbon haze (soot) production could act as a carbon-sink in the atmosphere, 


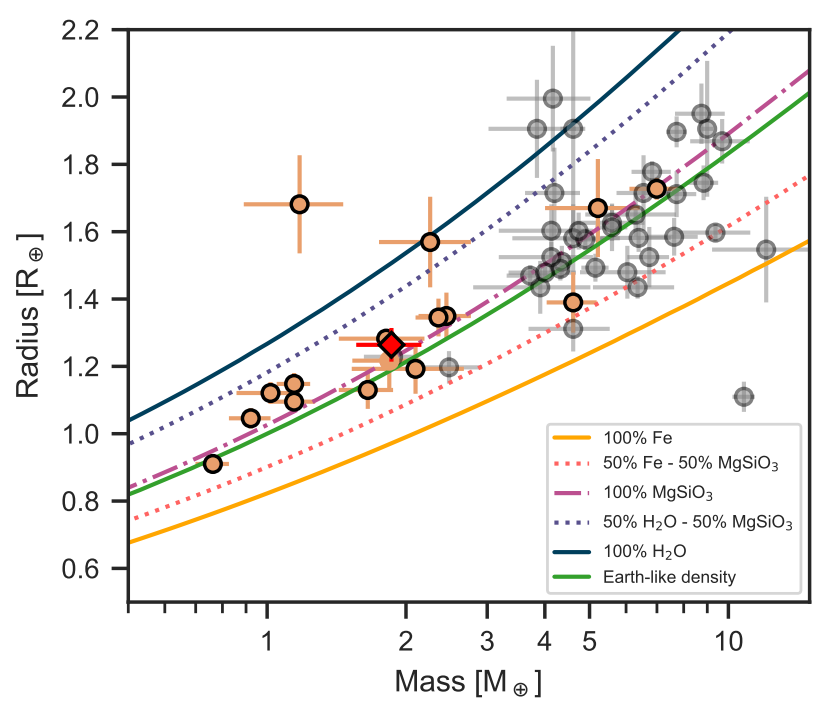

Fig. 10. Mass-radius diagram for small well characterised planets $\left(R<2 R_{\oplus}, \Delta M<30 \%\right.$ ) based on the TEPcat catalogue (Southworth 2011, visited on 14 April 2020). Planets orbiting stars with temperature $T_{\text {eff }}<4000 \mathrm{~K}$ are displayed in orange colour, while the rest is displayed as grey circles. GJ $3473 \mathrm{~b}$ is marked with a red diamond. For comparison, theoretical mass-radius relations from Zeng et al. $(2016,2019)$ are overlayed.

Table 5. Derived planetary parameters of GJ $3473 \mathrm{~b}$ and c based on the posteriors of the joint fit.

\begin{tabular}{lccl}
\hline \hline \multirow{2}{*}{ Parameter } & \multicolumn{2}{c}{ Posterior $^{(a)}$} & \multirow{2}{*}{ Units } \\
\cline { 2 - 3 } & \multicolumn{4}{c}{ GJ $3473 \mathrm{~b}$} & $\mathrm{GJ} 3473 \mathrm{c}$ & \\
\cline { 2 - 3 }$p=R_{\mathrm{p}} / R_{\star}$ & $0.03184_{-0.00067}^{+0.00069}$ & $\ldots$ & $\ldots$ \\
$b=\left(a / R_{\star}\right) \cos i_{\mathrm{p}}$ & $0.336_{-0.074}^{+0.066}$ & $\ldots$ & $\ldots$ \\
$a / R_{\star}$ & $9.39_{-0.21}^{+0.19}$ & $\ldots$ & $\ldots$ \\
$i_{p}$ & $87.95_{-0.45}^{+0.47}$ & $\ldots$ & $\mathrm{deg}$ \\
$u_{1, \mathrm{TESS}}$ & $0.26_{-0.18}^{+0.28}$ & $\ldots$ & $\ldots$ \\
$u_{2, \mathrm{TESS}}$ & $0.10_{-0.22}^{+0.28}$ & $\ldots$ & $\ldots$ \\
$t_{T}$ & $0.950_{-0.014}^{+0.015}$ & $\ldots$ & $\mathrm{h}$ \\
& Derived physical parameters ${ }^{(b)}$ & \\
$M_{\mathrm{p}}$ & $1.86_{-0.30}^{+0.30}$ & $\geq 7.41_{-0.86}^{+0.91}$ & $M_{\oplus}$ \\
$R_{\mathrm{p}}$ & $1.264_{-0.049}^{+0.050}$ & $\ldots$ & $R_{\oplus}$ \\
$\rho_{\mathrm{p}}$ & $5.03_{-0.93}^{+1.07}$ & $\ldots$ & $\mathrm{g} \mathrm{cm}{ }^{-3}$ \\
$g_{\mathrm{p}}$ & $11.4_{-2.0}^{+2.1}$ & $\ldots$ & $\mathrm{m} \mathrm{s}^{-2}$ \\
$a_{\mathrm{p}}$ & $0.01589_{-0.00062}^{+0.00062}$ & $0.0876_{-0.0034}^{+0.0035}$ & $\mathrm{au}$ \\
$T_{\mathrm{eq}}(c)$ & $773_{-15}^{+16}$ & $329.1_{-6.4}^{+6.6}$ & $\mathrm{~K}$ \\
$S$ & $59.4_{-4.5}^{+5.0}$ & $1.95_{-0.15}^{+0.17}$ & $S_{\oplus}$ \\
$\mathrm{ESM}^{(d)}$ & $6.8 \pm 0.3$ & $\ldots$ & $\ldots$ \\
\hline & & &
\end{tabular}

Notes. ${ }^{(a)}$ Error bars denote the $68 \%$ posterior credibility intervals. ${ }^{(b)} \mathrm{We}$ sample from a normal distribution for the stellar mass, stellar radius and stellar luminosity that is based on the results from Sect. 3. ${ }^{(c)}$ Assuming a zero Bond albedo. ${ }^{(d)}$ Emission spectroscopy metric (Kempton et al. 2018).

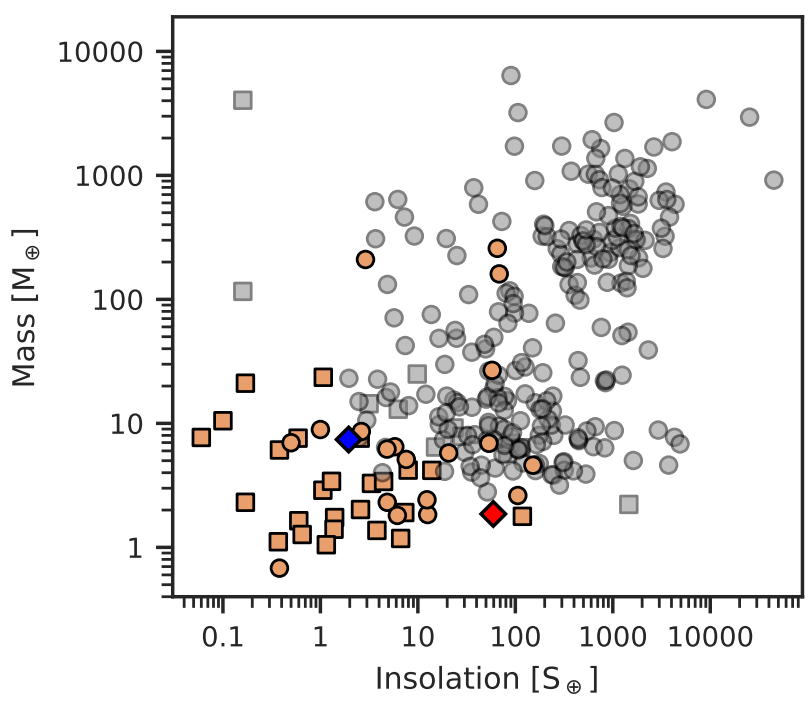

Fig. 11. Mass-insolation diagram for small RV planets based on the planetary systems composite data table of the exoplanetarchive. ipac.caltech.edu/ (visited on 28 August 2020). Planets orbiting stars with temperature $T_{\text {eff }}<4000 \mathrm{~K}$ are displayed in orange colour, while the rest is plotted as grey circles. Planets with a dynamical mass measurement are shown as circles and planets with only a minimum mass $(M \sin i)$ measurement with boxes. GJ $3473 \mathrm{~b}$ and $\mathrm{c}$ are marked with red and blue diamonds.

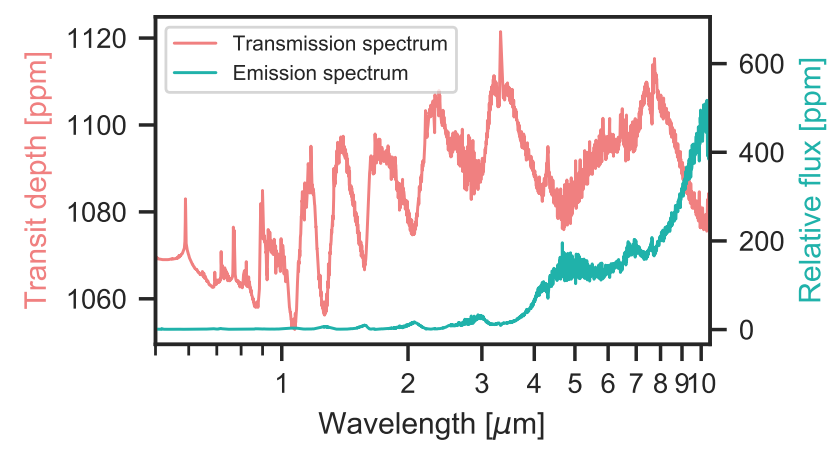

Fig. 12. Representative synthetic cloud free transmission and emission spectrum of GJ $3473 \mathrm{~b}$.

which might cause a reduced carbon-to-oxygen $(\mathrm{C} / \mathrm{O})$ ratio (e.g. Molaverdikhani et al. 2019b; Gao et al. 2020). While haze opacities tend to obscure the optical to NIR wavelength range, a reduced $\mathrm{C} / \mathrm{O}$ ratio could result in an enhancement of $\mathrm{CO}_{2}$ production. This causes a prominent feature at around $4.5 \mu \mathrm{m}$ (see e.g. Kawashima \& Ikoma 2019; Nowak et al. 2020). Atmospheres with higher metallicities are likely to lead to more prominent $\mathrm{CO}_{2}$ features (see e.g. Heng \& Lyons 2016; Molaverdikhani et al. 2019b; Nowak et al. 2020; Schlecker et al. 2020). Hence, this spectral feature appears to be a key feature to retrieve planetary atmosphere metallicities, which, in turn, helps us to understand the formation history of the planet and the stellar system.

The amplitudes of the transmission spectral features of GJ $3473 \mathrm{~b}$ are estimated to be around 10 to $40 \mathrm{ppm}$ for the discussed model. This poses a challenge for future observations of this planet through transmission spectroscopy. However, the relatively high temperature of this planet causes the emission spectral features at wavelengths longer than $\sim 3 \mu$ m to vary from tens of ppm in NIR to hundreds ppm in MIR wavelengths up to $4 \mu \mathrm{m}$. We calculate the emission spectroscopy metric (ESM), 

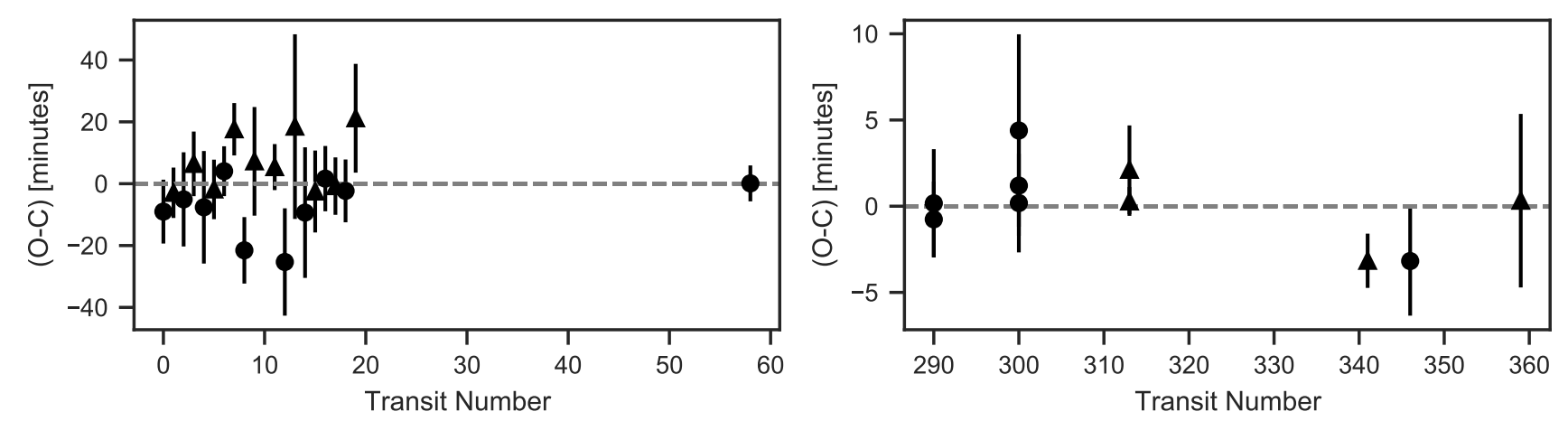

Fig. 13. TTVs measured for the transits of GJ $3473 \mathrm{~b}$ based on the results from the joint fit. Even transits are depicted as circles and odd transits as triangles. The observations corresponding to the transit numbers can be found in Table 1 .

based on Kempton et al. (2018), to be $6.8 \pm 0.3$, which is close to what Kempton et al. (2018) classify as high-quality atmospheric characterisation targets $(\mathrm{ESM}>7.5)$.

\subsection{GJ 3473 C}

Our RV modelling shows evidence for a second planet in the system. Its derived period is likely not linked to the stellar rotation period of $168 \mathrm{~d}$ as determined in Sect. 4.7. Furthermore, the analysis of a comprehensive set of activity indicators exhibits no signs of stellar activity at the period in question. The analysis of the $\mathrm{pEW}$ of the $\mathrm{H} \alpha$ line and the $\log R_{\mathrm{HK}}^{\prime}$ index describes GJ 3473 as a rather inactive star, which would contradict the relatively high RV amplitude of $\sim 3.8 \mathrm{~m} \mathrm{~s}^{-1}$ if the signal was attributed to activity (cf. Sect. 4.6). Furthermore, the signal is coherent for at least one year of observations and invariant with respect to the different instruments (see Sect. 4.8).

We therefore conclude that the $15.5 \mathrm{~d}$ signal in the RVs is caused by a second planet in the system, GJ 3473 c. The planet has a lower mass limit of $7.41_{-0.86}^{+0.91} M_{\oplus}$. Further physical parameters derived for this planet are shown in Table 5. No transit signals of GJ $3473 \mathrm{c}$ are found within the TESS data. An estimate of its bulk composition from theoretical models is not feasible because the derived mass places the planet just in the regime of the radius dichotomy between super-Earths and mini-Neptunes (e.g. Owen \& Wu 2013; Jin et al. 2014; Fulton et al. 2017; Zeng et al. 2017; Cloutier \& Menou 2020). However, the non-detection of transits is not unexpected when an orbit co-planar to GJ $3473 \mathrm{~b}$ $\left(i=87.95 \pm 0.47^{\circ}\right)$ is assumed. The minimum inclination for at least grazing transits at a separation of $0.0876 \pm 0.0035 \mathrm{au}$ from the host star would be $i>89.47^{\circ}$ considering a planet at the empirical upper radius limit for mini-Neptunes. At a distance of $8.66_{-0.13}^{+0.13} \times 10^{-2}$ au from the host star, GJ 3473 c receives $1.98_{-0.15}^{+0.17}$ times the stellar flux compared to Earth, which places it outside the inner boundary of the optimistic habitable zone, $1.49 S_{\oplus}>\mathrm{S}>0.22 S_{\oplus}$, as defined by Kopparapu et al. (2014). The planet therefore is a temperate super-Earth or mini-Neptune such as GJ 887 c (Jeffers et al. 2020), GJ 686 b (Lalitha et al. 2019; Affer et al. 2019), GJ 685 (Pinamonti et al. 2019) or GJ 581 c (Udry et al. 2007) (see Fig. 11).

\subsection{Comparison to synthetic planet populations}

We compare the planetary system of GJ 3473 with a synthetic $\mathrm{M}$ dwarf planet population from a core accretion model of planet formation (Burn et al., in prep.) to assess the frequency of such a configuration. There, planets like GJ $3473 \mathrm{~b}$ are relatively abundant and often accompanied by multiple other planets in the system. More than $10 \%$ of their synthetic systems contain systems with a combination of planets similar to GJ $3473 \mathrm{~b}$ and $\mathrm{c}$ with respect to their masses and periods. The systems with an architecture closest to GJ 3473 suggest a low bulk density for the outer planet, which can currently not be tested observationally. Another theoretical prediction from the core accretion paradigm is a higher frequency of distant companions for volatile-poor inner planets such as GJ 3473 b (Schlecker et al. 2020). While the current results do not demonstrate any clear evidence for planets beyond GJ 3473 c, further long-term monitoring is needed to probe the outer system.

\subsection{Search for transit timing variations}

The period ratio of the two planets $\left(P_{\mathrm{b}} \approx 1.20 \mathrm{~d}, P_{\mathrm{c}} \approx 15.5 \mathrm{~d}\right)$ does not suggest the presence of strong transit timing variations (TTV) for the transiting planet. However, we used juliet to perform a fit that only explores possible TTVs in the system. For this, we re-ran the joint fit but fixed all parameters to the results in Tables 3 and C.1 and added a TTV parameter for each transit (Gaussian distributed prior with 0 mean and a standard deviation of $0.03 \mathrm{~d}$, see the documentation of juliet for details). Although the results in Fig. 13 indicate TTVs up to $20 \mathrm{~min}$, the error bars are rather large. The main reason for this is the small transit depth of GJ 3473 b compared to the scatter of the data points (see Fig. 6). A GLS analysis of the TTVs reveals no significant periodicity that would indicate the presence of another massive planet in the system.

\section{Conclusions}

Here, we report the discovery of a planetary system around the M4.0 V dwarf GJ 3473 based on an extensive set of RV measurements from CARMENES, IRD, and HARPS, as well as space-based TESS photometry and photometric transit followup observations from LCOGT, MuSCAT, and MuSCAT2, and high-resolution images from Keck/NIRC2 and Gemini/NIRI. We confirm the planetary nature of GJ 3473 b (TOI-488.01) and present its detailed characterisation from a simultaneous fit of the RV and transit data. The short-period planet has a mass of $M_{\mathrm{b}}=1.86 \pm 0.30 M_{\oplus}$ and a radius of $R_{\mathrm{b}}=1.264 \pm 0.050 R_{\oplus}$, which yields a density that is consistent with a rocky composition. The planet complements the sample of small planets with mass and radius measurements better than $30 \%$ and contributes to the TESS mission's primary goal to measure the masses of 50 planets with radii smaller than $4 R_{\oplus}$. Its proximity 
to the host star makes GJ $3473 \mathrm{~b}$ attractive for thermal emission spectroscopy. Synthetic cloud-free emission spectra predict amplitudes of the transmission spectral features up to $100 \mathrm{~s} \mathrm{ppm}$ in the MIR.

The RV data show evidence for an additional, non-transiting planet in the system. GJ $3473 \mathrm{c}$ has a minimum mass of $M_{\mathrm{c}} \sin i=$ $7.41 \pm 0.91 M_{\oplus}$ and an orbital period of $P_{\mathrm{c}}=15.509 \pm 0.033 \mathrm{~d}$, which places it just outside the inner boundary of the habitable zone.

The planetary system of GJ 3473 is another multi-planet system discovered around an $\mathrm{M}$ dwarf with planets in the range of Earth-like masses to super-Earths and mini-Neptunes. A comparison with synthetic planet populations shows that systems similar to GJ 3473 may be relatively abundant and often host multiple planets. We therefore encourage further long-time monitoring of the system to find additional planets.

Acknowledgements. CARMENES is an instrument at the Centro Astronómico Hispano-Alemán de Calar Alto (CAHA, Almería, Spain). CARMENES was funded by the German Max-Planck-Gesellschaft (MPG), the Spanish Consejo Superior de Investigaciones Científicas (CSIC), the European Union through FEDER/ERF FICTS-2011-02 funds, and the members of the CARMENES Consortium (Max-Planck-Institut für Astronomie, Instituto de Astrofísica de Andalucía, Landessternwarte Königstuhl, Institut de Ciències de l'Espai, Insitut für Astrophysik Göttingen, Universidad Complutense de Madrid, Thüringer Landessternwarte Tautenburg, Instituto de Astrofísica de Canarias, Hamburger Sternwarte, Centro de Astrobiología and Centro Astronómico Hispano-Alemán), with additional contributions by the Spanish Ministry of Economy, the German Science Foundation through the Major Research Instrumentation Programme and Deutsche Forschungsgemeinschaft (DFG) Research Unit FOR2544 "Blue Planets around Red Stars", the Klaus Tschira Stiftung, the states of Baden-Württemberg and Niedersachsen, and by the Junt de Andalucía. Part of this work was funded by the Ministerio de Ciencia e Innovación via projects PID2019-109522GB-C51/2/3/4 and AYA2016-79425C3-1/2/3-P, (MEXT/)JSPS KAKENHI via grants 15H02063, JP17H04574 18H05442, JP18H01265, JP18H05439, 19J11805, JP19K14783, and 22000005 , JST PRESTO via grant JPMJPR1775, FCT/MCTES through national funds (PIDDAC, PTDC/FIS-AST/32113/2017) via grant UID/FIS/04434/2019, FEDER - Fundo Europeu de Desenvolvimento Regional through COMPETE2020 - Programa Operacional Competitividade e Internacionalização (POCI-010145-FEDER-032113), the FONDECYT project 3180063, Fundação para a Ciência e a Tecnologia through national funds and by FEDER through COMPETE2020 - Programa Operacional Competitividade e Internacionalização by these grants: UID/FIS/04434/2019; UIDB/04434/2020; UIDP/04434/2020; PTDC/FIS-AST/32113/2017 \& POCI-01-0145-FEDER-032113; PTDC/FISAST/28953/2017 \& POCI-01-0145-FEDER-028953. and the Internationa Graduate Program for Excellence in Earth-Space Science. Part of the data analysis was carried out on the Multi-wavelength Data Analysis System operated by the Astronomy Data Center, National Astronomical Observatory of Japan. Funding for the TESS mission is provided by NASA's Science Mission directorate. We acknowledge the use of public TESS Alert data from pipelines a the TESS Science Office and at the TESS Science Processing Operations Center This research has made use of the Exoplanet Follow-up Observation Program website, which is operated by the California Institute of Technology, under contract with the National Aeronautics and Space Administration under the Exoplanet Exploration Program. Resources supporting this work were provided by the NASA High-End Computing Program through the NASA Advanced Supercomputing Division at Ames Research Center for the production of the SPOC data products. This paper includes data collected by the TESS mission, which are publicly available from the Mikulski Archive for Space Telescopes This work makes use of observations from the LCOGT network. The analysis of this work has made use of a wide variety of public available software packages that are not referenced in the manuscript: Exo-Striker (Trifonov 2019), astropy (Astropy Collaboration 2018), scipy (Virtanen et al. 2020), numpy (Oliphant 2006), matplotlib (Hunter 2007), tqdm (da Costa-Luis 2019), pandas (The pandas development team 2020), seaborn (Waskom et al. 2020), lightkurve (Lightkurve Collaboration 2018) and PyFITS (Barrett et al. 2012).

\section{References}

Affer, L., Damasso, M., Micela, G., et al. 2019, A\&A, 622, A193 Alcock, C., Allsman, R., Alves, D. R., et al. 2000, ApJ, 542, 257

Almenara, J. M., DDorn, C., Bonfils, X., \& Udry, S. 2018, MNRAS, 478, 460
Alonso-Floriano, F. J., Morales, J. C., Caballero, J. A., et al. 2015, A\&A, 577, A128

Ambikasaran, S., Foreman-Mackey, D., Greengard, L., Hogg, D. W., \& O’Neil, M. 2015, IEEE Trans. Pattern Anal. Mach. Intell., 38, 252

Anglada-Escudé, G., Tuomi, M., Gerlach, E., et al. 2013, A\&A, 556, A126

Arenou, F., Luri, X., Babusiaux, C., et al. 2018, A\&A, 616, A17

Astropy Collaboration (Price-Whelan, A. M., et al.) 2018, AJ, 156, 123

Astudillo-Defru, N., Forveille, T., Bonfils, X., et al. 2017a, A\&A, 602, A88

Astudillo-Defru, N., Delfosse, X., Bonfils, X., et al. 2017b, A\&A, 600, A13

Astudillo-Defru, N., Cloutier, R., Wang, S. X., et al. 2020, A\&A, 636, A58

Baluev, R. V. 2008, MNRAS, 385, 1279

Baraffe, I., Chabrier, G., Barman, T. S., Allard, F., \& Hauschildt, P. H. 2003, A\&A, 402, 701

Barrett, P., Hsu, J. C., Hanley, C., et al. 2012, PyFITS: Python FITS Module, Astrophys. Source Code Libr., [record ascl:1207.009]

Batalha, N. E., Lewis, N. K., Line, M. R., Valenti, J., \& Stevenson, K. 2018, ApJ, 856, L34

Bauer, F. F., Zechmeister, M., Kaminski, A., et al. 2020, A\&A, 640, A50

Berta, Z. K., Irwin, J., Charbonneau, D., Burke, C. J., \& Falco, E. E. 2012, AJ, 144, 145

Berta-Thompson, Z. K., Irwin, J., Charbonneau, D., et al. 2015, Nature, 527, 204

Bitsch, B., Raymond, S. N., \& Izidoro, A. 2019, A\&A, 624, A109

Bluhm, P., Luque, R., Espinoza, N., et al. 2020, A\&A, 639, A132

Bonfils, X., Delfosse, X., Udry, S., et al. 2013, A\&A, 549, A109

Bonfils, X., Almenara, J.-M., Cloutier, R., et al. 2018, A\&A, 618, A142

Borucki, W. J. 2016, Rep. Prog. Phys., 79, 036901

Borucki, W. J., Koch, D., Basri, G., et al. 2010, Science, 327, 977

Brahm, R., Espinoza, N., Jordán, A., et al. 2019, AJ, 158, 45

Brown, T. M., Baliber, N., Bianco, F. B., et al. 2013, PASP, 125, 1031

Caballero, J. A., Guàrdia, J., López del Fresno, M., et al. 2016a, Proc. SPIE, 9910, 99100E

Caballero, J. A., Cortés-Contreras, M., Alonso-Floriano, F. J., et al. 2016b, in 19th Cambridge Workshop on Cool Stars, Stellar Systems, and the Sun, 148 Chabrier, G. 2003, PASP, 115, 763

Ciardi, D. R., Beichman, C. A., Horch, E. P., \& Howell, S. B. 2015, ApJ, 805, 16 Cifuentes, C., Caballero, J. A., Cortes-Contreras, M., et al. 2020, A\&A, 642, A115

Cloutier, R., \& Menou, K. 2020, AJ, 159, 211

Cloutier, R., Astudillo-Defru, N., Bonfils, X., et al. 2019, A\&A, 629, A111

Cloutier, R., Eastman, J. D., Rodriguez, J. E., et al. 2020a, AJ, 160, 3

Cloutier, R., Rodriguez, J. E., Irwin, J., et al. 2020b, AJ, 160, 22

Collins, K. A., Kielkopf, J. F., Stassun, K. G., \& Hessman, F. V. 2017, AJ, 153, 77

Colome, J., \& Ribas, I. 2006, IAU Spec. Session, 6, 11

Cortés-Contreras, M. 2016, PhD thesis, Universidad Complutense de Madrid, Spain

da Costa-Luis, C. 2019, J. Open Source Softw., 4, 1277

Delrez, L., Gillon, M., Triaud, A. H. M. J., et al. 2018, MNRAS, 475, 3577

Dittmann, J. A., Irwin, J. M., Charbonneau, D., et al. 2017, Nature, 544, 333

Dreizler, S., Crossfield, I. J. M., Kossakowski, D., et al. 2020, A\&A, submitted

Dressing, C. D., \& Charbonneau, D. 2015, ApJ, 807, 45

Eastman, J. D., Rodriguez, J. E., Agol, E., et al. 2019, PASP, submitted [arXiv:1907.09480]

Espinoza, N. 2018, Res. Notes Amer. Astron. Soc., 2, 209

Espinoza, N., \& Jordán, A. 2015, MNRAS, 450, 1879

Espinoza, N., Hartman, J. D., Bakos, G. A., et al. 2019a, AJ, 158, 63

Espinoza, N., Kossakowski, D., \& Brahm, R. 2019b, MNRAS, 490, 2262

Feng, F., Tuomi, M., \& Jones, H. R. A. 2017, MNRAS, 470, 4794

Foreman-Mackey, D., Agol, E., Angus, R., \& Ambikasaran, S. 2017, AJ, 154 220

Fukui, A., Narita, N., Tristram, P. J., et al. 2011, PASJ, 63, 287

Fulton, B. J., Petigura, E. A., Howard, A. W., et al. 2017, AJ, 154, 109

Fulton, B. J., Petigura, E. A., Blunt, S., \& Sinukoff, E. 2018, PASP, 130, 044504

Gagné, J., Mamajek, E. E., Malo, L., et al. 2018, AJ, 856, 23

Gaia Collaboration (Brown, A. G. A., et al.) 2018, A\&A, 616, A1

Gaidos, E., Mann, A. W., Kraus, A. L., \& Ireland, M. 2016, MNRAS, 457, 2877

Gao, P., Thorngren, D. P., Lee, G. K. H., et al. 2020, Nat. Astron., 4, 951

Giclas, H. L., Slaughter, C. D., \& Burnham, R. 1959, Lowell Observ. Bull., 4, 136

Gillon, M., Jehin, E., Lederer, S. M., et al. 2016, Nature, 533, 221

Gillon, M., Triaud, A. H. M. J., Demory, B.-O., et al. 2017, Nature, 542, 456

Gliese, W., \& Jahreiß, H. 1991, The Astronomical Data Center CD-ROM: Selected Astronomical Catalogs, Vol. I, NASA/Astronomical Data Center, Goddard Space Flight Center, Greenbelt, MD

Grimm, S. L., Demory, B.-O., Gillon, M., et al. 2018, A\&A, 613, A68

Hardegree-Ullman, K. K., Cushing, M. C., Muirhead, P. S., \& Christiansen, J. L. 2019, AJ, 158, 75

Hawley, S. L., Gizis, J. E., \& Reid, I. N. 1996, AJ, 112, 2799 
Heng, K., \& Lyons, J. R. 2016, ApJ, 817, 149

Henry, T. J., Jao, W., Subasavage, J. P., et al. 2006, AJ, 132, 2360

Hippke, M., \& Heller, R. 2019, A\&A, 623, A39

Hirano, T., Gaidos, E., Winn, J. N., et al. 2020, ApJ, 890, L27

Hodapp, K. W., Jensen, J. B., Irwin, E. M., et al. 2003, PASP, 115, 1388

Howard, A. W., Marcy, G. W., Bryson, S. T., et al. 2012, ApJS, 201, 15

Hunter, J. D. 2007, Comput. Sci. Eng., 9, 90

Husser, T.-O., Wende-von Berg, S., Dreizler, S., et al. 2013, A\&A, 553, A6

Jeffers, S. V., Schöfer, P., Lamert, A., et al. 2018, A\&A, 614, A76

Jeffers, S. V., Dreizler, S., Barnes, J. R., et al. 2020, Science, 368, 1477

Jeffreys, H. 1946, Proc. R. Soc. London Seri. A, 186, 453

Jenkins, J. M. 2002, ApJ, 575, 493

Jenkins, J. M., Twicken, J. D., McCauliff, S., et al. 2016, SPIE Conf. Ser., 9913, 99133E

Jenkins, J. S., Pozuelos, F. J., Tuomi, M., et al. 2019, MNRAS, 490, 5585

Jin, S., Mordasini, C., Parmentier, V., et al. 2014, ApJ, 795, 65

Kawashima, Y., \& Ikoma, M. 2019, ApJ, 877, 109

Kempton, E. M.-R., Bean, J. L., Louie, D. R., et al. 2018, PASP, 130, 114401

Kipping, D. M. 2013, MNRAS, 435, 2152

Kipping, D. M., Nesvorný, D., Buchhave, L. A., et al. 2014, ApJ, 784, 28

Kopparapu, R. K., Ramirez, R. M., SchottelKotte, J., et al. 2014, ApJ, 787, L29

Kossakowski, D., Espinoza, N., Brahm, R., et al. 2019, MNRAS, 490, 1094

Kostov, V. B., Schlieder, J. E., Barclay, T., et al. 2019, AJ, 158, 32

Kotani, T., Tamura, M., Suto, H., et al. 2018, SPIE, 10702, 37

Kreidberg, L. 2015, PASP, 127, 1161

Lafarga, M., Ribas, I., Lovis, C., et al. 2020, A\&A, 636, A36

Lalitha, S., Baroch, D., Morales, J. C., et al. 2019, A\&A, 627, A116

Li, J., Tenenbaum, P., Twicken, J. D., et al. 2019, PASP, 131, 024506

Lightkurve Collaboration (Cardoso, J. V. d. M., et al.) 2018, Lightkurve: Kepler and TESS time series analysis in Python

Lindegren, L., Hernández, J., Bombrun, A., et al. 2018, A\&A, 616, A2

Lissauer, J. J. 2007, ApJ, 660, L149

Lovis, C., \& Pepe, F. 2007, A\&A, 468, 1115

Luger, R., Sestovic, M., Kruse, E., et al. 2017, Nat. Astron., 1, 0129

Luque, R., Pallé, E., Kossakowski, D., et al. 2019, A\&A, 628, A39

Luyten, W. J. 1979, New Luyten catalogue of stars with proper motions larger than two tenths of an arcsecond; and first supplement; NLTT. (Minneapolis 1979); Label 12 = short description; Label 13 = documentation by Warren; Label 14 = catalogue

Madhusudhan, N. 2012, ApJ, 758, 36

Mann, A. W., Dupuy, T., Muirhead, P. S., et al. 2017, AJ, 153, 267

Marcy, G. W., Isaacson, H., Howard, A. W., et al. 2014, ApJS, 210, 20

Mason, B. D., Wycoff, G. L., Hartkopf, W. I., Douglass, G. G., \& Worley, C. E 2001, AJ, 122, 3466

Mayor, M., Pepe, F., Queloz, D., et al. 2003, The Messenger, 114, 20

McCully, C., Turner, M., Volgenau, N., et al. 2018, https://doi.org/10. 5281 /zenodo. 1257560

Ment, K., Dittmann, J. A., Astudillo-Defru, N., et al. 2019, AJ, 157, 32

Mills, S. M., \& Mazeh, T. 2017, ApJ, 839, L8

Molaverdikhani, K., Henning, T., \& Mollière, P. 2019a, ApJ, 873, 32

Molaverdikhani, K., Henning, T., \& Mollière, P. 2019b, ApJ, 883, 194

Molaverdikhani, K., Henning, T., \& Mollière, P. 2020, ApJ, 899, 53

Mollière, P., van Boekel, R., Dullemond, C., Henning, T., \& Mordasini, C. 2015, ApJ, 813, 47

Morales, J. C., Mustill, A. J., Ribas, I., et al. 2019, Science, 365, 1441

Mortier, A., \& Collier Cameron, A. 2017, A\&A, 601, A110

Mortier, A., Faria, J. P., Correia, C. M., Santerne, A., \& Santos, N. C. 2015, A\&A, 573, A101

Morton, T. D. 2015, Astrophysics Source Code Library [record ascl: 1503.010]

Mulders, G. D., Pascucci, I., \& Apai, D. 2015, ApJ, 798, 112

Narita, N., Fukui, A., Kusakabe, N., et al. 2015, J. Astron. Telesc. Instrum. Syst., 1,045001

Narita, N., Fukui, A., Kusakabe, N., et al. 2019, J. Astron. Telesc. Instrum. Syst., 5, 015001

Newton, E. R., Charbonneau, D., Irwin, J., et al. 2014, AJ, 147, 20

Newton, E. R., Irwin, J., Charbonneau, D., et al. 2017, ApJ, 834, 85

Nowak, G., Luque, R., Parviainen, H., et al. 2020, A\&A, 642, A173

Oliphant, T. E. 2006, A Guide to NumPy (Trelgol Publishing USA), 1

Owen, J. E., \& Wu, Y. 2013, ApJ, 775, 105

Parviainen, H. 2015, MNRAS, 450, 3233

Parviainen, H., Tingley, B., Deeg, H. J., et al. 2019, A\&A, 630, A89

Passegger, V. M., Reiners, A., Jeffers, S. V., et al. 2018, A\&A, 615, A6

Passegger, V. M., Schweitzer, A., Shulyak, D., et al. 2019, A\&A, 627, A161

Pinamonti, M., Sozzetti, A., Giacobbe, P., et al. 2019, A\&A, 625, A126

Pope, B. J. S., Parviainen, H., \& Aigrain, S. 2016, MNRAS, 461, 3399

Quirrenbach, A., Amado, P. J., Caballero, J. A., et al. 2014, Proc. SPIE, 9147, $91471 \mathrm{~F}$
Reid, I. N., Hawley, S. L., \& Gizis, J. E. 1995, AJ, 110, 1838

Reiners, A., Ribas, I., Zechmeister, M., et al. 2018, A\&A, 609, L5

Ricker, G. R., Winn, J. N., Vanderspek, R., et al. 2015, J. Astron. Telesc. Instrum. Syst., 1, 014003

Rogers, L. A. 2015, ApJ, 801, 41

Schlecker, M., Mordasini, C., Emsenhuber, A., et al. 2020, https://doi .org/ 10.1051/0004-6361/202038554

Schweitzer, A., Passegger, V. M., Cifuentes, C., et al. 2019, A\&A, 625, A68

Schöfer, P., Jeffers, S. V., Reiners, A., et al. 2019, A\&A, 623, A44

Service, M., Lu, J. R., Campbell, R., et al. 2016, PASP, 128, 095004

Shporer, A., Collins, K. A., Astudillo-Defru, N., et al. 2020, ApJ, 890, L7

Skrutskie, M. F., Cutri, R. M., Stiening, R., et al. 2006, AJ, 131, 1163

Smith, J. C., Stumpe, M. C., van Cleve, J. E., et al. 2012, PASP, 124, 1000

Southworth, J. 2010, MNRAS, 408, 1689

Southworth, J. 2011, MNRAS, 417, 2166

Speagle, J. S. 2020, MNRAS, 493, 3132

Stassun, K. G., Oelkers, R. J., Paegert, M., et al. 2019, AJ, 158, 138

Stumpe, M. C., Smith, J. C., van Cleve, J. E., et al. 2012, PASP, 124, 985

Stumpe, M. C., Smith, J. C., Catanzarite, J. H., et al. 2014, PASP, 126, 100

Tal-Or, L., Trifonov, T., Zucker, S., Mazeh, T., \& Zechmeister, M. 2019, MNRAS, 484, L8

The pandas development team. 2020, pandas-dev/pandas: Pandas

Tody, D. 1993, ASP Conf. Ser., 52, 173

Trifonov, T. 2019, Astrophysics Source Code Library [record ascl: 1906.004]

Trifonov, T., Kürster, M., Zechmeister, M., et al. 2018, A\&A, 609, A117

Trifonov, T., Tal-Or, L., Zechmeister, M., et al. 2020, A\&A, 636, A74

Trotta, R. 2008, Contemp. Phys., 49, 71

Twicken, J. D., Catanzarite, J. H., Clarke, B. D., et al. 2018, PASP, 130, 064502

Udry, S., Bonfils, X., Delfosse, X., et al. 2007, A\&A, 469, L43

Virtanen, P., Gommers, R., Oliphant Travis, E., et al. 2020, Nat. Methods, 17, 261

Waskom, M., Botvinnik, O., Ostblom, J., et al. 2020, mwaskom/seaborn: v0.10.1 (April 2020)

Zechmeister, M., \& Kürster, M. 2009, A\&A, 496, 577

Zechmeister, M., Reiners, A., Amado, P. J., et al. 2018, A\&A, 609, A12

Zeng, L., Sasselov, D. D., \& Jacobsen, S. B. 2016, ApJ, 819, 127

Zeng, L., Jacobsen, S. B., \& Sasselov, D. D. 2017, Res. Notes Amer. Astron. Soc., 1,32

Zeng, L., Jacobsen, S. B., Sasselov, D. D., et al. 2019, Proc. Natl. Acad. Sci. USA, 116, 9723

Zhu, Z., Hartmann, L., Nelson, R. P., \& Gammie, C. F. 2012, Res. Notes Amer. Astron. Soc., 746, 110

1 Landessternwarte, Zentrum für Astronomie der Universität Heidelberg, Königstuhl 12, 69117 Heidelberg, Germany e-mail: j.kemmer@lsw.uni-heidelberg.de

2 Max-Planck-Institut für Astronomie, Königstuhl 17, 69117 Heidelberg, Germany

3 Centro de Astrobiología (CSIC-INTA), ESAC, Camino bajo del castillo s/n, 28692 Villanueva de la Cañada, Madrid, Spain

4 Instituto de Astrofísica de Andalucía (IAA-CSIC), Glorieta de la Astronomía s/n, 18008 Granada, Spain

5 Departamento de Matemática y Físíca Aplicadas, Universidad Católica de la Santísima Concepción, Alonso de Rivera 2850 , Concepción, Chile

6 Univ. Grenoble Alpes, CNRS, IPAG, 38000 Grenoble, France

7 Caltech/IPAC-NASA Exoplanet Science Institute, $770 \mathrm{~S}$. Wilson Avenue, Pasadena, CA 91106, USA

8 Center for Astrophysics I Harvard \& Smithsonian, 60 Garden Street, Cambridge, MA 02138, USA

9 Space Telescope Science Institute, 3700 San Martin Drive, Baltimore, MD 21218, USA

10 Department of Earth and Planetary Science, Graduate School of Science, The University of Tokyo, 7-3-1 Hongo, Bunkyo-ku, Tokyo 113-0033, Japan

11 Departamento de Astrofísica, Universidad de La Laguna, 38206 La Laguna, Tenerife, Spain

12 Department of Earth and Planetary Sciences, Tokyo Institute of Technology, 2-12-1 Ookayama, Meguro-ku, Tokyo 152-8551, Japan

13 NASA Ames Research Center, Moffett Field, CA 94035, USA

14 Department of Physics and Kavli Institute for Astrophysics and Space Research, Massachusetts Institute of Technology, Cambridge, MA 02139, USA 
15 Komaba Institute for Science, The University of Tokyo, 3-8-1 Komaba, Meguro, Tokyo 153-8902, Japan

16 JST, PRESTO, 3-8-1 Komaba, Meguro, Tokyo 153-8902, Japan

17 Astrobiology Center, 2-21-1 Osawa, Mitaka, Tokyo 181-8588, Japan

${ }^{18}$ Instituto de Astrofísica de Canarias (IAC), 38205 La Laguna, Tenerife, Spain

${ }^{19}$ Institut für Astrophysik, Georg-August-Universität, Friedrich-HundPlatz 1, 37077 Göttingen, Germany

${ }^{20}$ Institut de Ciències de l'Espai (ICE, CSIC), Campus UAB, Can Magrans s/n, 08193 Bellaterra, Spain

${ }^{21}$ Institut d'Estudis Espacials de Catalunya (IEEC), 08034 Barcelona, Spain

22 NASA Goddard Space Flight Center, 8800 Greenbelt Road, Greenbelt, MD 20771, USA

${ }^{23}$ Department of Earth, Atmospheric and Planetary Sciences, Massachusetts Institute of Technology, Cambridge, MA 02139, USA

24 Department of Aeronautics and Astronautics, MIT, 77 Massachusetts Avenue, Cambridge, MA 02139, USA

25 Department of Astrophysical Sciences, Princeton University, 4 Ivy Lane, Princeton, NJ 08544, USA

26 Observatoire de Genève, Université de Genève, $51 \mathrm{Ch}$. des Maillettes, 1290 Sauverny, Switzerland

27 George Mason University, 4400 University Drive, Fairfax, VA, 22030 USA

28 European Space Agency, ESTEC, Keplerlaan 1, 2201 AZ Noordwijk, The Netherlands

29 Department of Astronomy, The University of Tokyo, 7-3-1 Hongo, Bunkyo-ku, Tokyo 113-0033, Japan

${ }^{30}$ Department of Physics, United States Air Force Academy, Colorado, CO 80840 , USA

${ }^{31}$ European Southern Observatory, Alonso de Córdova 3107, Vitacura, Región Metropolitana, Chile

32 Instituto de Astrofísica e Ciências do Espaço, Universidade do Porto, CAUP, Rua das Estrelas, 4150-762 Porto, Portugal
${ }^{33}$ Centro Astronómico Hispano-Alemán, Observatorio de Calar Alto, Sierra de los Filabres, 04550 Gérgal, Spain

34 Department of Astronomy, Tsinghua University, Beijing 100084, PR China

35 Department of Astronomy and Astrophysics, University of California, Santa Cruz, 1156 High St. Santa Cruz, CA 95064, USA

36 Observatori Astronómic Albanyá, Camí de Bassegoda s/n, Albanyá 17733, Girona, Spain

37 Subaru Telescope, 650 N. Aohoku Place, Hilo, HI 96720, USA

38 Thüringer Landessternwarte Tautenburg, Sternwarte 5, 07778 Tautenburg, Germany

39 University of Hawaii, Institute for Astronomy, 640 N. Aohoku Place, Hilo, HI 96720, USA

40 National Astronomical Observatory of Japan, 2-21-1 Osawa, Mitaka, Tokyo 181-8588, Japan

${ }^{41}$ Okayama Observatory, Kyoto University, 3037-5 Honjo, Kamogatacho, Asakuchi, Okayama 719-0232, Japan

42 Deutsches Zentrum für Luft und Raumfahrt, Institut für Planetenforschung Rutherfordstrasse 2, 12489 Berlin, Germany

43 Department of Astronomical Science, The Graduated University for Advanced Studies, SOKENDAI, 2-21-1, Osawa, Mitaka, Tokyo 1818588 Japan

44 Tokyo University of Agriculture and Technology, 3-8-1, Saiwai-cho, Fuchu, Tokyo 183-0054, Japan

45 U.S. Naval Observatory, 3450 Massachusetts Avenue NW, Washington, D.C. 20392, USA

46 Department of Astronomy, Institute for Astrophysical Research, Boston University, 725 Commonwealth Ave. Room 514, Boston, MA 02215, USA

47 Departamento de Física e Astronomia, Faculdade de Ciências, Universidade do Porto, Rua do Campo Alegre, 4169-007 Porto, Portugal

${ }^{48}$ Patashnick Voorheesville Observatory, Voorheesville, NY 12186, USA

49 SETI Institute, Mountain View, CA 94043, USA 


\section{Appendix A: Known transiting planets with precise mass measurements around $M$ dwarfs}

Table A.1. Small transiting planets with precise masses around $\mathrm{M}$ dwarfs.

\begin{tabular}{|c|c|c|c|c|c|}
\hline Name & Alternative name & $\begin{array}{c}\text { Radius } \\
{\left[R_{\oplus}\right]} \\
\end{array}$ & & $\begin{array}{l}\text { Mass } \\
{\left[M_{\oplus}\right]}\end{array}$ & Reference \\
\hline GJ $3473 \mathrm{~b}^{(a, b)}$ & G $50-16$ b & $1.264 \pm 0.050$ & 1.86 & \pm 0.30 & This work \\
\hline LP 729-54 $\mathrm{b}^{(a, b)}$ & LTT $3780 \mathrm{~b}$ & $1.35 \pm 0.06$ & 2.34 & \pm 0.24 & Nowak et al. (2020); Cloutier et al. (2020a) \\
\hline TOI-1235 $\mathrm{b}^{(a, b)}$ & TYC 4384-1735-1 b & $1.69 \pm 0.08$ & 5.9 & \pm 0.6 & Bluhm et al. (2020); Cloutier et al. (2020b) \\
\hline GJ $357 \mathrm{~b}^{(a, b)}$ & LHS $2157 \mathrm{~b}$ & $1.217 \pm 0.084$ & 1.84 & \pm 0.31 & Luque et al. (2019); Jenkins et al. (2019) \\
\hline GJ $1252 \mathrm{~b}^{(a)}$ & $\mathrm{L} 210-70 \mathrm{~b}$ & $1.193 \pm 0.074$ & 2.10 & \pm 0.58 & Shporer et al. (2020) \\
\hline L $98-59 \mathrm{c}^{(a)}$ & TOI-175.01 & $1.35 \pm 0.07$ & 2.42 & \pm 0.35 & Cloutier et al. (2019); Kostov et al. (2019) \\
\hline $\mathrm{L} 98-59 \mathrm{~d}^{(a)}$ & TOI-175.01 & $1.57 \pm 0.14$ & 2.31 & \pm 0.46 & Cloutier et al. (2019); Kostov et al. (2019) \\
\hline L $168-9 b^{(a)}$ & CD-608051 b & $1.39 \pm 0.09$ & 4.60 & \pm 0.58 & Astudillo-Defru et al. (2020) \\
\hline Kepler-138 c & KOI-314.2 & $1.67 \pm 0.15$ & 5.2 & \pm 1.3 & Almenara et al. (2018); Kipping et al. (2014); Mann et al. (2017) \\
\hline Kepler-138 d & KOI-314.3 & $1.68 \pm 0.15$ & 1.17 & \pm 0.30 & Almenara et al. (2018); Kipping et al. (2014); Mann et al. (2017) \\
\hline GJ 1132 b & LTT $3758 b$ & $1.130 \pm 0.057$ & 1.66 & \pm 0.23 & Bonfils et al. (2018); Berta-Thompson et al. (2015) \\
\hline LHS $1140 \mathrm{~b}$ & GJ 3053 b & $1.727 \pm 0.033$ & 6.99 & \pm 0.89 & Ment et al. (2019); Dittmann et al. (2017) \\
\hline LHS 1140 c & GJ 3053 c & $1.282 \pm 0.024$ & 1.81 & \pm 0.39 & Ment et al. (2019) \\
\hline TRAPPIST-1 b & 2MUCD $12171 \mathrm{~b}$ & $1.121 \pm 0.033$ & 1.017 & \pm 0.16 & Grimm et al. (2018); Delrez et al. (2018); Gillon et al. (2016) \\
\hline TRAPPIST- $1 \mathrm{c}$ & 2MUCD $12171 \mathrm{c}$ & $1.095 \pm 0.031$ & 1.156 & \pm 0.15 & Grimm et al. (2018); Delrez et al. (2018); Gillon et al. (2016) \\
\hline TRAPPIST-1 d & 2MUCD $12171 \mathrm{~d}$ & $0.784 \pm 0.023$ & 0.297 & $7 \pm 0.039$ & Grimm et al. (2018); Delrez et al. (2018); Gillon et al. (2017) \\
\hline TRAPPIST-1 e & 2MUCD $12171 \mathrm{e}$ & $0.910 \pm 0.027$ & 0.772 & $2 \pm 0.079$ & Grimm et al. (2018); Delrez et al. (2018); Gillon et al. (2017) \\
\hline TRAPPIST-1 f & 2MUCD $12171 \mathrm{f}$ & $1.046 \pm 0.030$ & 0.934 & $4 \pm 0.095$ & Grimm et al. (2018); Delrez et al. (2018); Gillon et al. (2017) \\
\hline TRAPPIST-1 g & 2MUCD $12171 \mathrm{~g}$ & $1.148 \pm 0.033$ & 1.148 & \pm 0.098 & Grimm et al. (2018); Delrez et al. (2018); Gillon et al. (2017) \\
\hline TRAPPIST-1 h & 2MUCD $12171 \mathrm{~h}$ & $0.773 \pm 0.027$ & 0.331 & \pm 0.056 & Grimm et al. (2018); Delrez et al. (2018); Luger et al. (2017) \\
\hline
\end{tabular}

Notes. ${ }^{(a)}$ Planets discovered by TESS. ${ }^{(b)}$ Target stars in the CARMENES guaranteed time observations survey (Quirrenbach et al. 2014; Reiners et al. 2018). The table is based on TEPCat (Southworth 2011, visited on 15 July 2020) and shows the known transiting planets with radii smaller than $2 R_{\oplus}$ and mass determinations to a precision better than $30 \%$ in orbits around stars with temperatures lower than $4000 \mathrm{~K}$. The first reference always denotes the source of the properties. 


\section{Appendix B: Priors for juliet}

Table B.1. Priors used for juliet in the joint fit of transits and RV.

\begin{tabular}{|c|c|c|c|}
\hline Parameter & Prior & Units & Description \\
\hline \multicolumn{4}{|c|}{ Stellar parameters } \\
\hline$\rho_{\star}$ & $\mathcal{N}(10520.0,836.2)$ & $\mathrm{kg} \mathrm{m}^{-3}$ & Stellar density \\
\hline \multicolumn{4}{|c|}{ Planetary parameters } \\
\hline$P_{\mathrm{b}}$ & $\mathcal{N}(1.1980004,0.000009)$ & $\mathrm{d}$ & Period of the transiting planet \\
\hline$t_{0, \mathrm{~b}}$ & $\mathcal{N}(2458492.2041,0.0015)$ & $\mathrm{d}$ & Time of transit centre of the transiting planet \\
\hline$r_{1, \mathrm{~b}}$ & $\mathcal{N}(0.55,0.15)$ & $\ldots$ & Parametrisation for $\mathrm{p}$ and $\mathrm{b}$ \\
\hline$r_{2, \mathrm{~b}}$ & $\mathcal{N}(0.0318,0.0021)$ & $\ldots$ & Parametrisation for $\mathrm{p}$ and $\mathrm{b}$ \\
\hline$K_{\mathrm{b}}$ & $\mathcal{N}(2.4,1.5)$ & $\mathrm{m} \mathrm{s}^{-1}$ & Radial-velocity semi-amplitude of the transiting planet \\
\hline$\sqrt{e_{\mathrm{b}}} \sin \omega_{\mathrm{b}}$ & fixed $(0)$ & $\ldots$ & Parametrisation for $e$ and $\omega$. \\
\hline$\sqrt{e_{\mathrm{b}}} \cos \omega_{\mathrm{b}}$ & fixed(0) & $\ldots$ & Parametrisation for $e$ and $\omega$. \\
\hline$P_{\mathrm{c}}$ & $\mathcal{N}(15.51,0.16)$ & $\mathrm{d}$ & Period of the second RV signal \\
\hline$t_{0, \mathrm{c}}$ & $\mathcal{N}(2458575.7,1.5)$ & $\mathrm{d}$ & Time of transit centre of the second RV signal \\
\hline$K_{\mathrm{c}}$ & $\mathcal{N}(3.7,1.5)$ & $\mathrm{m} \mathrm{s}^{-1}$ & Radial-velocity semi-amplitude of the second RV signal \\
\hline$\sqrt{e_{\mathrm{c}}} \sin \omega_{\mathrm{c}}$ & fixed $(0)$ & $\ldots$ & Parametrisation for $e$ and $\omega$. \\
\hline$\sqrt{e_{\mathrm{c}}} \cos \omega_{\mathrm{c}}$ & fixed $(0)$ & & Parametrisation for $e$ and $\omega$. \\
\hline \multicolumn{4}{|c|}{ Instrument parameters CARMENES, HARPS, IRD } \\
\hline$\mu$ & $\mathcal{U}(-10,10)$ & $\mathrm{m} \mathrm{s}^{-1}$ & Instrumental offset \\
\hline$\sigma$ & $\mathcal{U}(0,10)$ & $\mathrm{m} \mathrm{s}^{-1}$ & Jitter term \\
\hline \multicolumn{4}{|c|}{ Instrument parameters TESS } \\
\hline$q_{1}$ & $\mathcal{U}(0,1)$ & $\ldots$ & Quadratic limb-darkening parametrisation \\
\hline$q_{2}$ & $\mathcal{U}(0,1)$ & $\ldots$ & Quadratic limb-darkening parametrisation \\
\hline mdilution & fixed(1) & $\ldots$ & Dilution factor \\
\hline mflux & $\mathcal{N}(0.0, .01)$ & ppm & Instrumental offset \\
\hline$\sigma$ & $\mathcal{U}(1,500)$ & ppm & Jitter term \\
\hline \multicolumn{4}{|c|}{ Instrument parameters MuSCAT2 } \\
\hline$q_{1}$ & $\mathcal{U}(0,1)$ & $\ldots$ & Linear limb-darkening parametrisation \\
\hline mdilution & fixed(1) & $\cdots$ & Dilution factor \\
\hline mflux & $\mathcal{N}(0.0, .01)$ & ppm & Instrumental offset \\
\hline$\sigma$ & $\mathcal{U}(1,500)$ & ppm & Jitter term \\
\hline \multicolumn{4}{|c|}{ Instrument parameters MuSCAT, LCOGT } \\
\hline$q_{1}$ & $\mathcal{U}(0,1)$ & $\ldots$ & Linear limb-darkening parametrisation \\
\hline mdilution & fixed(1) & $\cdots$ & Dilution factor \\
\hline mflux & $\mathcal{N}(0.0, .01)$ & ppm & Instrumental offset \\
\hline$\sigma$ & $\mathcal{U}(1,500)$ & ppm & Jitter term \\
\hline$\theta_{0}$ & $\mathcal{U}(-100,100)$ & $\ldots$ & Linear airmass detrending coefficient \\
\hline
\end{tabular}

Notes. The prior labels, $\mathcal{U}$ and $\mathcal{N}$, represent uniform and normal distributions, respectively.

Table B.2. Priors used with juliet for the determination of the rotation period.

\begin{tabular}{llcl}
\hline \hline Parameter & Prior & Units & Description \\
\hline & & Instrument parameters Mearth, TJO \\
mdilution & fixed(1) & $\ldots$ & Dilution factor \\
mflux & $\mathcal{N}(0.0,1 e 5)$ & ppm & Instrumental offset \\
$\sigma$ & $\mathcal{J}(1 e-5,1 e 5)$ & ppm & Jitter term \\
& & GP parameters & (individual) Mearth, TJO \\
GP- $\sigma$ & $\mathcal{J}(1 e-8,1 e 8)$ & ppm & GP amplitude \\
GP- $\Gamma$ & $\mathcal{J}(1 e-2,1 e 2)$ & $\ldots$ & GP amplitude of the sine-squared component \\
& & GP parameters & shared) Mearth, TJO \\
GP- $\alpha$ & $\mathcal{J}(1 e-10,1)$ & $\mathrm{d}^{-2}$ & GP inverse length scale of the exponential component \\
GP- $P_{\text {rot }}$ & $\mathcal{U}(2,200)$ & d & GP rotation period of the quasi-periodic component \\
\hline
\end{tabular}

Notes. The prior labels, $\mathcal{U}$ and $\mathcal{N}$, represent uniform, and normal distributions. $\mathcal{J}$ is the log-uniform Jeffrey's distribution (Jeffreys 1946). 


\section{Appendix C: Continuation of the posteriors}

Table C.1. Posteriors of the joint fit for the different instrumental parameters.

\begin{tabular}{|c|c|c|c|c|c|}
\hline Parameter & Posterior $^{(a)}$ & Units & Parameter & Posterior $^{(a)}$ & Units \\
\hline \multicolumn{3}{|c|}{ TESS } & \multicolumn{3}{|c|}{ MuSCAT $_{r} 18$ Jan. 2020} \\
\hline$q_{1}$ & $0.17_{-0.12}^{+0.25}$ & $\cdots$ & $q_{1}$ & $0.867_{-0.172}^{+0.09}$ & $\ldots$ \\
\hline$q_{2}$ & $0.35_{-0.24}^{+0.33}$ & $\ldots$ & $\sigma$ & $385_{-143}^{+76}$ & ppm \\
\hline$\sigma$ & $34_{-22}^{+31}$ & ppm & $\theta_{0}$ & $0.0054_{-0.0011}^{+0.0011}$ & $\ldots$ \\
\hline \multicolumn{3}{|c|}{ LCO McD z $_{s} 19$ Mar. 2019} & \multicolumn{3}{|c|}{ MuSCAT $_{z}$ s 18 Jan. 2020} \\
\hline$q_{1}$ & $0.49_{-0.30}^{+0.29}$ & $\cdots$ & $q_{1}$ & $0.147_{-0.098}^{+0.150}$ & $\ldots$ \\
\hline$\sigma$ & $437_{-78}^{+42}$ & ppm & $\sigma$ & $440.0_{-55.0}^{+38.0}$ & $\mathrm{ppm}$ \\
\hline$\theta_{0}$ & $-0.00302_{-0.00058}^{+0.00058}$ & $\cdots$ & $\theta_{0}$ & $0.00346_{-0.00069}^{+0.00067}$ & $\cdots$ \\
\hline \multicolumn{3}{|c|}{ MuSCAT2 $_{i} 21$ Dec. 2019} & \multicolumn{3}{|c|}{ LCO CTIO } \\
\hline$q_{1}$ & $0.77_{-0.26}^{+0.16}$ & $\cdots$ & $q_{1}$ & $0.873_{-0.158}^{+0.085}$ & $\ldots$ \\
\hline$\sigma$ & $113_{-72}^{+104}$ & ppm & $\sigma$ & $492.9_{-9.2}^{+4.9}$ & ppm \\
\hline \multicolumn{3}{|c|}{ MuSCAT2 $2_{z s} 21$ Dec. 2019} & $\theta_{0}$ & $0.0015_{-0.00017}^{+0.00017}$ & $\cdots$ \\
\hline$q_{1}$ & $0.57_{-0.31}^{+0.26}$ & $\cdots$ & \multicolumn{3}{|c|}{ LCO CTIO ${ }_{i p} 27$ Feb. 2020} \\
\hline$\sigma$ & $155_{-100}^{+136}$ & ppm & $q_{1}$ & $0.52_{-0.30}^{+0.28}$ & $\ldots$ \\
\hline \multicolumn{3}{|c|}{ MuSCAT2 $_{i} 2$ Jan. 2020} & $\sigma$ & $482_{-22}^{+12}$ & ppm \\
\hline$q_{1}$ & $0.57_{-0.31}^{+0.27}$ & $\cdots$ & $\theta_{0}$ & $0.00243_{-0.00018}^{+0.00017}$ & $\cdots$ \\
\hline$\sigma$ & $114_{-72}^{+99}$ & ppm & \multicolumn{3}{|c|}{ LCO SAAO ${ }_{z s} 13$ Mar. 2020} \\
\hline \multicolumn{3}{|c|}{ MuSCAT2 ${ }_{r} 2$ Jan. 2020} & $q_{1}$ & $0.60_{-0.33}^{+0.26}$ & $\ldots$ \\
\hline$q_{1}$ & $0.54_{-0.31}^{+0.28}$ & $\cdots$ & $\sigma$ & $413_{-98}^{+56}$ & ppm \\
\hline$\sigma$ & $121_{-77}^{+109}$ & ppm & $\theta_{0}$ & $-0.00086_{-0.00071}^{+0.0007}$ & $\cdots$ \\
\hline \multicolumn{3}{|c|}{ MuSCAT2 $2_{z s} 2$ Jan. 2020} & & & \\
\hline $\begin{array}{l}q_{1} \\
\sigma\end{array}$ & $\begin{array}{c}0.78_{-0.23}^{+0.15} \\
109_{-69}^{+90}\end{array}$ & $\begin{array}{l}\cdots \\
\mathrm{ppm}\end{array}$ & & & \\
\hline
\end{tabular}

Notes. ${ }^{(a)}$ Error bars denote the $68 \%$ posterior credibility intervals. 